\title{
THE KEPLER-10 PLANETARY SYSTEM REVISITED BY HARPS-N: A HOT ROCKY WORLD AND A SOLID NEPTUNE-MASS PLANET*
}

\author{
Xavier Dumusque ${ }^{1,16}$, Aldo S. Bonomo ${ }^{2}$, Raphä̈lle D. Haywood ${ }^{3}$, Luca Malavolta ${ }^{4,5}$, Damien SÉgransan $^{6}$, \\ Lars A. Buchhave ${ }^{1,7}$, Andrew Collier Cameron ${ }^{3}$, David W. Latham ${ }^{1}$, Emilio Molinari ${ }^{8,9}$, Francesco Pepe $^{6}$, \\ Stéphane Udry $^{6}$, David Charbonneau ${ }^{1}$, Rosario Cosentino ${ }^{8}$, Courtney D. Dressing $^{1}$, Pedro Figueira $^{10}$, \\ Aldo F. M. Fiorenzano ${ }^{8}$, Sara Gettel ${ }^{1}$, Avet Harutyunyan ${ }^{8}$, Keith Horne ${ }^{3}$, Mercedes Lopez-Morales $^{1}$, \\ Christophe Lovis $^{6}$, Michel MaYor ${ }^{6}$, Giusi Micela ${ }^{11}$, FAtemeh Motalebi $^{6}$, Valerio Nascimbeni ${ }^{5}$, David F. Phillips ${ }^{1}$, \\ Giampaolo Piotto $^{4,5}$, Don Pollacco ${ }^{12}$, Didier Queloz ${ }^{6,13}$, Ken Rice ${ }^{14}$, Dimitar Sasselov ${ }^{1}$, Alessandro Sozzetti $^{2}$, \\ ANDREW SZENTGYORGYI ${ }^{1}$, AND Chris WATSON ${ }^{15}$ \\ ${ }^{1}$ Harvard-Smithsonian Center for Astrophysics, 60 Garden Street, Cambridge, MA 02138, USA; xdumusque@cfa.harvard.edu \\ ${ }^{2}$ INAF - Osservatorio Astrofisico di Torino, via Osservatorio 20, I-10025 Pino Torinese, Italy \\ ${ }^{3}$ SUPA, School of Physics \& Astronomy, University of St. Andrews, North Haugh, St. Andrews Fife KY16 9SS, UK \\ ${ }^{4}$ Dipartimento di Fisica e Astronomia "Galileo Galilei," Universita'di Padova, Vicolo dell'Osservatorio 3, I-35122 Padova, Italy \\ ${ }^{5}$ INAF - Osservatorio Astronomico di Padova, Vicolo dell'Osservatorio 5, I-35122 Padova, Italy \\ ${ }^{6}$ Observatoire Astronomique de l'Université de Genève, $51 \mathrm{ch}$. des Maillettes, CH-1290 Versoix, Switzerland \\ ${ }^{7}$ Centre for Star and Planet Formation, Natural History Museum of Denmark, University of Copenhagen, DK-1350 Copenhagen, Denmark \\ ${ }^{8}$ INAF - Fundacin Galileo Galilei, Rambla Jos Ana Fernandez Prez 7, E-38712 Brea Baja, Spain \\ ${ }^{9}$ INAF - IASF Milano, via Bassini 15, I-20133, Milano, Italy \\ ${ }^{10}$ Centro de Astrofisica, Universidade do Porto, Rua das Estrelas, 4150-762 Porto, Portugal \\ ${ }^{11}$ INAF - Osservatorio Astronomico di Palermo, Piazza del Parlamento 1, I-90124 Palermo, Italy \\ ${ }^{12}$ Department of Physics, University of Warwick, Gibbet Hill Road, Coventry CV4 7AL, UK \\ ${ }^{13}$ Cavendish Laboratory, J J Thomson Avenue, Cambridge CB3 OHE, UK \\ ${ }^{14}$ SUPA, Institute for Astronomy, Royal Observatory, University of Edinburgh, Blackford Hill, Edinburgh EH9 3HJ, UK \\ ${ }_{15}$ Astrophysics Research Centre, School of Mathematics and Physics, Queens University, Belfast, UK \\ Received 2014 March 20; accepted 2014 May 28; published 2014 June 24
}

\begin{abstract}
Kepler-10b was the first rocky planet detected by the Kepler satellite and confirmed with radial velocity follow-up observations from Keck-HIRES. The mass of the planet was measured with a precision of around 30\%, which was insufficient to constrain models of its internal structure and composition in detail. In addition to Kepler-10b, a second planet transiting the same star with a period of 45 days was statistically validated, but the radial velocities were only good enough to set an upper limit of $20 M_{\oplus}$ for the mass of Kepler-10c. To improve the precision on the mass for planet b, the HARPS-N Collaboration decided to observe Kepler-10 intensively with the HARPS-N spectrograph on the Telescopio Nazionale Galileo on La Palma. In total, 148 high-quality radial-velocity measurements were obtained over two observing seasons. These new data allow us to improve the precision of the mass determination for Kepler-10b to $15 \%$. With a mass of $3.33 \pm 0.49 M_{\oplus}$ and an updated radius of $1.47_{-0.02}^{+0.03} R_{\oplus}$, Kepler-10b has a density of $5.8 \pm 0.8 \mathrm{~g} \mathrm{~cm}^{-3}$, very close to the value predicted by models with the same internal structure and composition as the Earth. We were also able to determine a mass for the 45-day period planet Kepler-10c, with an even better precision of $11 \%$. With a mass of $17.2 \pm 1.9 M_{\oplus}$ and radius of $2.35_{-0.04}^{+0.09} R_{\oplus}$, Kepler-10c has a density of $7.1 \pm 1.0 \mathrm{~g} \mathrm{~cm}^{-3}$. Kepler-10c appears to be the first strong evidence of a class of more massive solid planets with longer orbital periods.
\end{abstract}

Key words: planetary systems - stars: individual (Kepler-10 KOI-072 KIC 11904151) - stars: statistics techniques: photometric - techniques: spectroscopic

Online-only material: color figures, machine-readable table

\section{INTRODUCTION}

Transiting planets enable a rich variety of opportunities to explore planetary astrophysics, ranging from the determination of bulk densities to studies of system architectures and atmospheric compositions, and even climate and weather. However, for most of these studies, a critical first step is the determination of a dynamical mass that confirms and characterizes the planetary nature of the candidate. This mass, together with the size

\footnotetext{
* Based on observations made with the Italian Telescopio Nazionale Galileo (TNG) operated on the island of La Palma by the Fundacin Galileo Galilei of the INAF (Istituto Nazionale di Astrofisica) at the Spanish Observatorio del Roque de los Muchachos of the Instituto de Astrofisica de Canarias.

${ }^{16}$ Swiss National Science Foundation Fellow.
}

of the planet determined from the light curve, yields the bulk density of the planet.

The importance of determining dynamical masses for small planets, either by Doppler spectroscopy or transit-time variations (TTVs) or a photo-dynamical analysis, was brought into focus by the results for the five small inner planets transiting Kepler-11, ranging in size from 1.8 to 4.2 times the diameter of the Earth (Lissauer et al. 2011). The masses derived from TTVs eventually led to densities ranging from 1.7 down to $0.58 \mathrm{~g} \mathrm{~cm}^{-3}$ (Lissauer et al. 2013), much less than the Earth's bulk density of $5.5 \mathrm{~g} \mathrm{~cm}^{-3}$, presumably due to extended atmospheres of hydrogen and helium. It became clear that a better understanding of the occurrence rate for planets similar to the Earth would require density determinations for a significant population of small planets. 
CoRot-7b was the first small planet that looked like it might be dense enough to be rocky (Queloz et al. 2009; Léger et al. 2009). However, determining its dynamical mass was quite challenging, because strong stellar activity was perturbing the radial velocities (RVs) of the host star. This inspired work on ways to reduce the impact of activity-induced RV signals: prewhitening by the rotation period and harmonics (Dumusque et al. 2012; Boisse et al. 2011; Queloz et al. 2009), correlation with activity indicators (Boisse et al. 2009), velocity differences using multiple observations during the same night (Hatzes et al. 2010), correlation with photometric variations (Aigrain et al. 2012), and treating stellar activity as correlated noise (Feroz \& Hobson 2013, R. D. Haywood et al., in preparation). This work is finally leading to a consensus that CoRoT-7b has a density consistent with a rocky planet. Moreover, learning how to correct the CoRoT photometry and RV measurements for stellar activity is now paying off for other cases (e.g., Pepe et al. 2013; Howard et al. 2013; Dumusque et al. 2012).

Kepler-10b is in many ways a twin of CoRoT-7b, in terms of size and orbital period, but has the advantage of transiting an old quiet star. Still, the mass reported in the discovery paper, $4.56 \pm 1.3 M_{\oplus}$, has a precision not much better than three sigma (Batalha et al. 2011), which is well short of the accuracy needed to distinguish a rocky planet from a water world, nominally $10 \%$, as we argue below. The discovery paper (Batalha et al. 2011) also reported the detection of a second transiting planet candidate with a period of 45 days and radius of $2.23 R_{\oplus}$ in the Kepler photometry. However, no orbital motion at this period was detected in the observed RVs, and only an upper limit of $20 M_{\oplus}$ could be placed on the mass. With more Kepler data and simultaneous Spitzer Space Telescope photometry, Fressin et al. (2011) reanalyzed the Kepler-10 system and statistically validated the planetary nature of Kepler-10c with BLENDER (Torres et al. 2011). This result was not a surprise, because planet candidates in multi-transit systems are much less likely to be false positives than candidates in single-transit systems (Latham et al. 2011).

Kepler-36 has provided the most accurate mass for a confirmed rocky planet, based on the extreme TTVs observed in the Kepler photometry for this system, which boasts two transiting planets in orbits close to a 6:7 resonance (Carter et al. 2012). This is an example of a new approach, a photo-dynamical analysis that uses the rich information available when three or more bodies all eclipse or transit each other in compact orbits. However, this is a bizarre system in which the neighboring planets have densities that differ by a factor of eight, and it is not clear how the system formed, or how much longer it will survive. But, photo-dynamical analysis is a powerful path to detailed masses and radii for those extremely rare systems with mutual events and continuous high-quality light curves such as those provided by Kepler.

Kepler-78b is the smallest transiting planet with a welldetermined mass (radius of $1.16 R_{\oplus}$ and mass of $1.86 M_{\oplus}$ ), implying a density very similar to that of the Earth, but with large uncertainties (Pepe et al. 2013; Howard et al. 2013). This recent success was enabled by the extremely short orbital period of $8.5 \mathrm{hr}$, implying a molten surface facing the host star and most likely a very different history of formation compared to the Earth.

Another important recent result is the heroic effort to determine masses for a few dozen small planet candidates using HIRES (Marcy et al. 2014), with positive detections for about two dozen. Even though the uncertainties on the masses of these objects are typically one or two sigma, this is still good enough to verify that they are planets because of the strong constraints provided by the photometric ephemerides. As a population, many of them are likely to be rocky, but the results are not good enough to characterize any individual planet in detail.

The HARPS-N Collaboration established the goal of measuring masses and radii with an accuracy sufficient to constrain models of the internal structure and composition of individual small planets, focusing on those with compact atmospheres. For example, an accuracy of nominally $10 \%$ or better in mass and $5 \%$ or better in radius is needed to distinguish rocky planets with iron cores from those with extensive water content, according to recent models (Zeng \& Sasselov 2013).

At the start of HARPS-N science operations in 2012 August, Kepler-10b looked like the best target for pursuing this goal. It had already been confirmed as a rocky planet, with a mass of $4.56 M_{\oplus}$, a radius of $1.42 R_{\oplus}$, and a density of $8.8 \mathrm{~g} \mathrm{~cm}^{-3}$ (Batalha et al. 2011). In addition, the stellar parameters of Kepler-10 are well constrained by asteroseismology (FogtmannSchulz et al. 2014), which gives us an unprecedented precision on the radius of Kepler-10b. The mass determination was based on 40 precise RV measurements obtained with KeckHIRES, and the uncertainty in the density of nearly $30 \%$ was dominated by the uncertainty in the mass. Over the 2012 and 2013 observing seasons, we accumulated nearly four times as many RVs for Kepler-10 (with similar precision, see Pepe et al. 2013; Howard et al. 2013). In this paper, we present the analysis of these new data, which allow us to improve the uncertainty of the mass for Kepler-10b by about a factor of two, and to determine precisely the mass of Kepler-10c for the first time.

\section{OBSERVATIONS AND DATA REDUCTION}

We monitored the RV variation of Kepler-10 with the HARPS-N spectrograph installed on the 3.57-m Telescopio Nazionale Galileo at the Spanish Observatorio del Roque de los Muchachos, La Palma Island, Spain (Cosentino et al. 2012). This instrument is an updated version of the original HARPS planet hunter installed on the 3.6-m telescope at the European Southern Observatory on La Silla, Chile (Mayor et al. 2003). Just like its older brother, the HARPS-N instrument is an ultrastable fiber-fed high-resolution $(R=115,000)$ optical echelle spectrograph optimized for the measurement of very precise RVs. The use of a more modern monolithic $4 \mathrm{k} \times 4 \mathrm{k}$ CCD enclosed in a more temperature stable cryostat, and the use of octagonal fibers for a better scrambling of the incoming light fed into the spectrograph should improve the precision of the instrument compared to HARPS. By observing standard stars of known constant RV during the first year of operation, we estimated the RV precision to be of at least $1 \mathrm{~m} \mathrm{~s}^{-1}$ when not limited by photon noise. When observing fainter stars, we expect a RV precision of $1.2 \mathrm{~m} \mathrm{~s}^{-1}$ to be achieved in a 1-h exposure on a slowly rotating late-G or K-type dwarf with $m_{V}=12$.

Scientific operations began at HARPS-N in 2012 August. Over the first two observing seasons, we obtained 157 RV measurements of Kepler-10. Four observations that were obtained during bad weather conditions had very low signal to noise $(\mathrm{S} / \mathrm{N}$, $<10$ ) and were rejected. During the first year of operation, in 2012, half of the CCD stopped working and the stellar spectra were only recorded on half of the echelle orders. With only a subset of the lines used to derive the RV using the cross correlation technique, it is not clear if the data taken with half of the chip can be used or not. We therefore decided to reject those five points that were taken before the CCD was replaced. This paper 
Table 1

HARPS-N RV Measurements

\begin{tabular}{|c|c|c|c|c|c|c|c|}
\hline $\begin{array}{c}\text { BJD }-2400000 \\
\text { (days) }\end{array}$ & $\begin{array}{c}\mathrm{RV} \\
\left(\mathrm{km} \mathrm{s}^{-1}\right)\end{array}$ & $\begin{array}{l}\text { RV Error } \\
\left(\mathrm{km} \mathrm{s}^{-1}\right)\end{array}$ & $\begin{array}{l}\text { FWHM } \\
\left(\mathrm{km} \mathrm{s}^{-1}\right)\end{array}$ & $\begin{array}{l}\text { FWHM Error } \\
\qquad\left(\mathrm{km} \mathrm{s}^{-1}\right)\end{array}$ & $\begin{array}{l}\log \left(R_{H K}^{\prime}\right) \\
\quad(\operatorname{dex})\end{array}$ & $\begin{array}{c}\log \left(R_{H K}^{\prime}\right) \text { Error } \\
(\text { dex })\end{array}$ & $\begin{array}{c}\mathrm{S} / \mathrm{N} \\
550 \mathrm{~nm}\end{array}$ \\
\hline 56072.682384 & -98.742550 & 0.001760 & -0.001413 & 0.003948 & -4.9939 & 0.0212 & 56.20 \\
\hline 56072.704768 & -98.742890 & 0.001860 & -0.010146 & 0.004183 & -4.9569 & 0.0250 & 53.40 \\
\hline 56087.575720 & -98.741520 & 0.002190 & -0.004506 & 0.004982 & -4.9606 & 0.0394 & 45.00 \\
\hline 56087.596901 & -98.735480 & 0.001980 & -0.012499 & 0.004465 & -4.9903 & 0.0334 & 48.50 \\
\hline 56103.661644 & -98.740400 & 0.002390 & -0.013195 & 0.005499 & -4.9856 & 0.0346 & 38.70 \\
\hline
\end{tabular}

(This table is available in its entirety in a machine-readable form in the online journal. A portion is shown here for guidance regarding its form and content.)

therefore presents $148 \mathrm{RV}$ observations of Kepler-10, which are listed in Table 1.

Kepler-10 has a Kepler magnitude $K p=10.96\left(m_{V}=11.16\right)$, so exposure times of 30 minutes were required to reach a $\mathrm{S} / \mathrm{N}$ adequate for high RV precision. Scaling from the expected precision for faint stars given in the first paragraph of this section, 30 minutes of exposure on the slowly rotating G-type dwarf Kepler-10 should yield a precision of $1.12 \mathrm{~m} \mathrm{~s}^{-1}$. Looking at the data, the best precision we could get on a single measurement was $1.16 \mathrm{~m} \mathrm{~s}^{-1}$ for an $\mathrm{S} / \mathrm{N}$ of 80 at $550 \mathrm{~nm}$. However, due to not always optimal conditions, the average error on the entire RV set is $1.63 \mathrm{~m} \mathrm{~s}^{-1}$ for an average $\mathrm{S} / \mathrm{N}$ of 59 at $550 \mathrm{~nm}$. This precision is two times smaller than the RV semiamplitude of Kepler-10b (Batalha et al. 2011), and was expected to be roughly the same as the semi-amplitude of Kepler-10c, assuming that planet has the same mass as the similar-sized planet Kepler-68b (2.31 $R_{\oplus}, 8.3 M_{\oplus}$; Gilliland et al. 2013).

The data exhibit a small RV offset between the measurements taken with the old and the new CCD mainly because of the different charge transfer efficiency of the detectors. This offset will be considered as a free parameter when fitting the RVs.

\section{STELLAR PROPERTIES}

Stellar parameters for Kepler-10 have been determined using a combined spectrum with $\mathrm{S} / \mathrm{N}$ of 750 at $550 \mathrm{~nm}$, resulting from the co-addition of all the individual spectra with $S / N \geqslant 30$, after dividing by the blaze function and correcting for the barycentric velocity.

Equivalent widths (EWs) were measured using a modification of the ARES code (Sousa et al. 2007), which provides an estimate of the error in the continuum determination and the root mean square of the residuals of the Gaussian fit. These values, together with the FWHM of each line, were used to reject lines with poorly measured EWs. Weak lines with EWs $\leqslant 5 \mathrm{~m} \AA$ were excluded from the analysis because of sensitivity to continuum determination. In addition, lines with $\mathrm{EW} \geqslant 110$ $\mathrm{m} \AA$ were also not considered because those lines depart from Gaussian shape.

Atmospheric parameters were determined using the 2013 version of the local thermodynamic equilibrium code MOOG (Sneden 1973) and the Kurucz model atmosphere grid ${ }^{17}$ calculated with the new opacity distribution function (ODFNEW; Castelli \& Kurucz 2004; Kurucz 1992). Atmospheric models were interpolated with a software developed by A. McWilliam and I. Ivans and provided by C. Sneden.

We adopted the line list from Sousa et al. (2011), but the oscillator strength values $\log g f$ were re-determined by

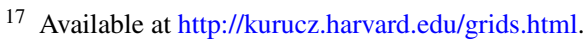

inverse analysis of their solar EWs using an Iron abundance of $\log \epsilon(\mathrm{Fe})=7.52$ (and the other photospheric parameters unchanged), to be consistent with the solar value used in the calculation of the model atmosphere grid and by MOOG in the abundance computation.

Atmospheric parameters were derived in a classical way. Effective temperature $T_{\text {eff }}$ was adjusted until there was no dependance of individual $\mathrm{Fe}$ I abundances with the excitation potential (EP) of the lines. The microturbulence velocity $\xi_{\mathrm{t}}$ was determined by requiring that the Fe I abundance be independent of the reduced equivalent widths $(\mathrm{REW}=\mathrm{EW} / \lambda)$. Surface gravity $\log g_{\star}$ was estimated by imposing the ionization equilibrium, i. e., by forcing agreement between the abundances derived from Fe I and Fe II lines. We cycled between each parameter iteratively until convergence was reached. To make sure that our determination was not influenced by the initial guess of the atmospheric parameters, we tested our results against several different input values, finding no significant difference in the derived atmospheric parameters.

Internal errors were estimated by considering the dispersion around the mean $\sigma(\mathrm{Fe} I)$ of $\log \epsilon(\mathrm{Fe} I)$ and its effect on the parameter determination. For $\sigma_{T_{\text {eff }}}$ we considered the variation in temperature resulting from a slope equal to the ratio between $\sigma(\mathrm{Fe} \mathrm{I})$ and the range in EP, and similarly for $\sigma_{\xi_{\mathrm{t}}}$ using the range in REW. The dependance of the atmospheric parameters on temperature was evaluated by repeating the analysis with the effective temperature fixed at $T_{\text {eff }} \pm \sigma_{T_{\text {eff }}}$, following Cayrel et al. (2004).

To derive the final atmospheric parameters, we fixed the value of $\log g_{\star}$ to 4.34, as derived in the recent asteroseismology study of Fogtmann-Schulz et al. (2014). This choice was made because the gravity of solar-type stars is known to be better constrained by asteroseismology, and because the value derived from EW analysis, $\log g_{\star}=4.38 \pm 0.8$ is fully in agreement. With this value fixed, the final atmospheric parameters for Kepler10 are $T_{\text {eff }}=5721 \pm 26 \mathrm{~K},[\mathrm{Fe} / \mathrm{H}]=-0.14 \pm 0.02$, and $\xi_{\mathrm{t}}=1.10 \pm 0.05 \mathrm{~km} \mathrm{~s}^{-1}$. This result was obtained using 191 Fe I and Fe II lines. The same analysis performed on several individual spectra returned values consistent within the errors, i. e., co-addition of spectra did not introduce any systematic effect. For comparison, the analysis on the Sun using the HARPS reflection spectrum of Ganymede provided by Sousa et al. (2007) results in $T_{\text {eff }}=5781 \pm 22 \mathrm{~K}, \log g_{\star}=4.45 \pm 0.06$, $\xi_{\mathrm{t}}=1.03 \pm 0.04 \mathrm{~km} \mathrm{~s}^{-1},[\mathrm{Fe} / \mathrm{H}]=0.00 \pm 0.02$, which are perfectly consistent with the canonical values for the Sun.

To double-check our derived atmospheric parameters, we also used the SPC code (Buchhave et al. 2012) to derive these parameters with a different method. The final values we found with this technique, fixing $\log g_{\star}$ to 4.34 , are 

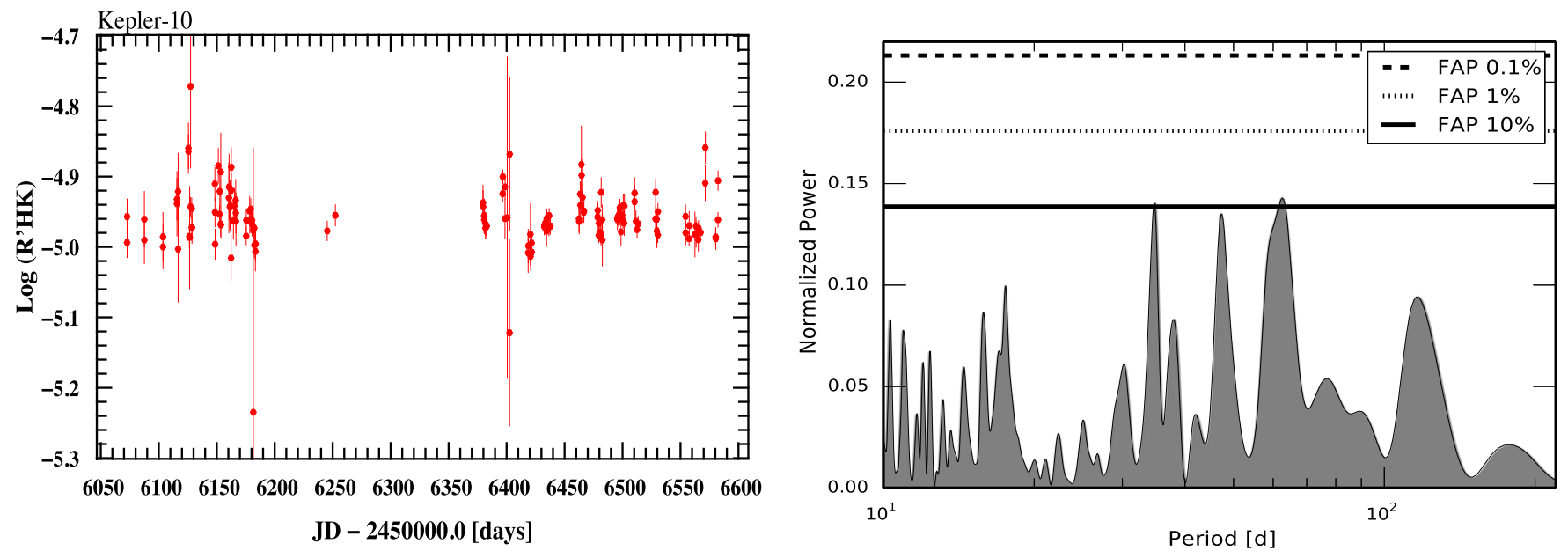

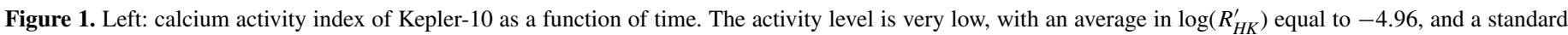
error of 0.04 dex. Right: periodogram of the calcium activity index. No clear variation in the activity index is detected as no peaks exhibit a FAP lower than $10 \%$.

(A color version of this figure is available in the online journal.)

Table 2

Kepler-10 Stellar and Atmospheric Parameters

\begin{tabular}{lcc}
\hline \hline Parameters & Values & References \\
\hline Mass, $M_{\star}\left(M_{\odot}\right)$ & $0.910 \pm 0.021$ & $\mathrm{~A}$ \\
Radius, $R_{\star}\left(R_{\odot}\right)$ & $1.065 \pm 0.009$ & $\mathrm{~A}$ \\
Stellar density, $\bar{\rho}_{\star}\left(\mathrm{g} \mathrm{cm}^{-3}\right)$ & $1.068 \pm 0.004$ & $\mathrm{~B}$ \\
Age $(\mathrm{Gyr})$ & $10.6_{-1.3}^{+1.5}$ & $\mathrm{~A}$ \\
Distance $(\mathrm{pc})$ & $173 \pm 27$ & $\mathrm{C}$ \\
Luminosity, $L_{\star}\left(L_{\odot}\right)$ & $1.004 \pm 0.059$ & $\mathrm{C}$ \\
Absolute $V$ magnitude, $M_{V}(\mathrm{mag})$ & $4.746 \pm 0.063$ & $\mathrm{C}$ \\
Magnitude, $m_{V}(\mathrm{mag})$ & 11.157 & $\mathrm{D}$ \\
Color index $(B-V)$ & 0.622 & $\mathrm{D}$ \\
Effective temperature, $T_{\mathrm{eff}}(\mathrm{K})$ & $5708 \pm 28$ & $\mathrm{~A}$ \\
Surface gravity, $\log g_{\star}$ & $4.344 \pm 0.004(\mathrm{fixed})$ & $\mathrm{A}$ \\
Microturbulence velocity, $\xi_{\mathrm{t}}\left(\mathrm{km} \mathrm{s}^{-1}\right)$ & $1.07 \pm 0.05$ & $\mathrm{~A}$ \\
Metallicity, $[\mathrm{Fe} / \mathrm{H}]$ & $-0.15 \pm 0.04$ & $\mathrm{~A}$ \\
Activity index, $\left\langle\log \left(R_{H K}^{\prime}\right)\right\rangle$ & -4.96 & $\mathrm{~A}$ \\
Projected rotation, $v$ sin $i\left(\mathrm{~km} \mathrm{~s}^{-1}\right)$ & $0.6 \pm 0.5-2.04 \pm 0.34$ & $\mathrm{~A}$ \\
\hline
\end{tabular}

Notes. Two values can be found for the $v \sin i$. The first one has been derived with SPC (Buchhave et al. 2012) by fitting synthetic spectra to the observed spectra. This type of analysis is not sensitive to $v \sin i$ values below $\sim 2 \mathrm{~km} \mathrm{~s}^{-1}$ because of the limit imposed by the spectrograph resolution. The other value is derived using the full width at half-maximum of the cross correlation function (see Section 4.2.2). The error bars represent a $1 \sigma$ uncertainty on the parameters. References. A: this work, B: Fogtmann-Schulz et al. (2014), C: Fressin et al. (2011), D: Everett et al. (2012).

$T_{\text {eff }}=5695 \pm 50 \mathrm{~K},[\mathrm{Fe} / \mathrm{H}]=-0.16 \pm 0.08$, and $v \sin i=$ $0.6 \pm 0.5 \mathrm{~km} \mathrm{~s}^{-1}$. These results are consistent with the preceding analysis, which provides confidence in the derived atmospheric parameters.

The adopted $T_{\text {eff }}$ and $[\mathrm{Fe} / \mathrm{H}]$ are the averages of the values obtained independently with the two aforementioned methods, i.e., $T_{\text {eff }}=5708 \pm 28 \mathrm{~K}$ and $[\mathrm{Fe} / \mathrm{H}]=-0.15 \pm 0.04$. The mass, radius, surface gravity, and age of Kepler-10 (see Table 2) were afterward determined by comparing the asteroseismic stellar density $\rho_{\star}$ as measured by Fogtmann-Schulz et al. (2014), the $T_{\text {eff }}$, and $[\mathrm{Fe} / \mathrm{H}]$ with the Yonsei-Yale evolutionary tracks (Torres et al. 2012; Sozzetti et al. 2007; Demarque et al. 2004) and by performing the same chi-square minimization as in Santerne et al. (2011). Uncertainties on stellar parameters were estimated through 5000 Monte Carlo simulations by assuming independent Gaussian errors on $\rho_{\star}, T_{\text {eff }}$, and $[\mathrm{Fe} / \mathrm{H}]$. Stellar parameters and their $1 \sigma$ errors are listed in Table 2 and are practically identical to those derived by Fogtmann-Schulz et al. (2014), as expected, because our effective temperature and stellar metallicity are consistent within $1 \sigma$ with the values found by these authors. No more iterations to re-determine the atmospheric parameters were required because the derived stellar surface gravity, i.e., $\log g_{\star}=4.344 \pm 0.004$, is equal to the previously fixed value.

The center-of-mass velocity of Kepler-10 relative to the solar system barycenter is $-98.7 \mathrm{~km} \mathrm{~s}^{-1}$. This is an unusually large RV that was already noticed in Batalha et al. (2011). Given this

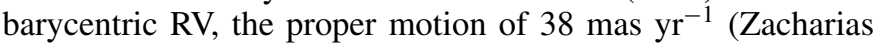
et al. 2009), and the estimated distance of $173 \mathrm{pc}$ (Fressin et al. 2011), the probability of the star being a member of the thick disk is $96 \%$ (Soubiran \& Girard 2005). This is consistent with the old age of the star, i.e., $10.6_{-1.3}^{+1.5}$ Gyr. Stars of the thick disc are normally more metal poor than Kepler-10, like for example WASP-21 (Bouchy et al. 2010) with a $[\mathrm{Fe} / \mathrm{H}]=-0.46$. However the separation in metallicity is not such a clear-cut argument (Adibekyan et al. 2013, and references therein).

\section{ANALYSIS OF THE RADIAL VELOCITY DATA}

This section is dedicated to the detection of the orbital motion induced by Kepler-10b and $\mathrm{c}$ in the HARPS-N RV data, and the best estimation of their masses. We first discuss the activity of the star and its rotational period, as these parameters can give us important information for the selection of the best model to use when fitting the data. Indeed, significant stellar activity will induce RV variations, and in this case, a suitable model should be considered.

\subsection{Kepler-10 Stellar Activity and Induced Radial Velocity Variations}

Kepler-10 is a very old main sequence star, estimated to be 10.6 Gyr old, which should imply a low activity level. When measuring the $\mathrm{Ca}$ II $\mathrm{HK}$ chromospheric activity index (hereafter calcium activity index) of Kepler-10 (Noyes et al. 1984), which is the best activity proxy for solar type stars, we find an average value of $\log \left(R_{H K}^{\prime}\right)$ equal to -4.96 , with a standard error of 0.04 dex (see Figure 1). This average is near the lower end of 
the solar value, which varies between -5 and -4.8 along its activity cycle. It is possible that we just observed the star during its minimum activity phase, which represents $\sim 30 \%$ of the solar cycle. However, during the four years of Kepler photometry, we do not see any strong variation similar to that induced by typical solar active regions when the Sun is active (see last following paragraph). The study of the activity index points to a star quieter than the Sun, which is compatible with its old age. With this low activity level, we do not expect the RVs to be significantly affected (Dumusque et al. 2011a).

To estimate the RV variations induced by activity for Kepler10 , we compared the amplitude of the variations seen in the Kepler photometry with that expected from active region simulations. Excluding quarters 4 and 17 because they were not detrended by the PDCMAP reduction pipeline (Smith et al. 2012), and quarter 9 because of instrumental systematics, the maximum peak-to-peak variation in the photometry is less than $500 \mathrm{ppm}$. Assuming that this variation is due to activity and not to instrumental systematics, and assuming a star with no limb darkening, a $500 \mathrm{ppm}$ variation in photometry would be induced by a dark spot with a filling factor of $0.05 \%$. According to Boisse et al. (2012), a 1\% dark spot would induce a RV semiamplitude of $35 \mathrm{~m} \mathrm{~s}^{-1}$ for a $v \sin i$ of $3 \mathrm{~km} \mathrm{~s}^{-1}$. Scaling from this value, this spot would induce a $1.75 \mathrm{~m} \mathrm{~s}^{-1}$ semi-amplitude. Considering a more sophisticated model that includes quadratic limb-darkening, convective blueshift suppression in active regions, and a more realistic $v \sin i$ of $2 \mathrm{~km} \mathrm{~s}^{-1}$ (see Table 2), this spot would have a filling factor of $0.09 \%$, and would induce a peak-to-peak RV variation of $1.1 \mathrm{~m} \mathrm{~s}^{-1}$ (X. Dumusque et al., in preparation). If instead we assume that a plage is inducing this $500 \mathrm{ppm}$ photometric variation, its size would be much larger, $1.4 \%$, and it would induce a RV peak to-peak variation of $5 \mathrm{~m} \mathrm{~s}^{-1}$. However a plage would also strongly affect the FWHM of the cross correlation function by a peak-to-peak variation of $20 \mathrm{~m} \mathrm{~s}^{-1}$ that is not observed (X. Dumusque et al., in preparation). In conclusion, the variations observed in the Kepler-10 photometry, if due to activity, would be induced by the presence of spots, and would correspond to a maximum RV peak-to-peak modulation of $\sim 1 \mathrm{~m} \mathrm{~s}^{-1}$, lower than the RV error bars (see Section 2). We therefore do not expect the RVs to be dominated by an activity signal, which is consistent with the low activity index of $\log \left(R_{H K}^{\prime}\right)=-4.96$ that is measured for the star.

\subsection{Kepler-10 Rotational Period}

In this section, we estimate the rotational period of Kepler-10 using several different techniques: activity and stellar age estimations, projected rotational velocity, and the Kepler photometry.

\subsubsection{Kepler-10 Rotational Period Estimation with Activity Index and Stellar Age}

The activity level and rotational period are strongly correlated for main sequence stars. Indeed, stellar activity is generated through the stellar magnetic dynamo, the strength of which appears to scale with rotation velocity (Montesinos et al. 2001; Noyes et al. 1984). Both stellar activity and rotation are observed to decay with age (Mamajek \& Hillenbrand 2008; Barnes 2007; Pace \& Pasquini 2004; Soderblom et al. 1991; Wilson 1963). In the course of their evolution, solar-type stars lose angular momentum via magnetic braking due to coupling with their stellar wind (Mestel 1968; Weber \& Davis 1967; Schatzman 1962).
With an age of $10.6 \mathrm{Gyr}$, Kepler-10 is a very old main sequence star, which should imply a rotational period longer than the Sun, and therefore a lower activity level. We saw in the preceding section that the activity level of Kepler-10 is very low, with an average value of $\log \left(R_{H K}^{\prime}\right)=-4.96$. With this average activity value and a color index $(B-V)=0.622$ (Everett et al. 2012), we can estimate a rotational period of $21.9 \pm 3.0$ days using the empirical relation of Mamajek \& Hillenbrand (2008). The age of $3.7 \mathrm{Gyr}$ given by this empirical relation is much younger than the value derived with asteroseismology. However gyrochronology relations are not well constrained for old mainsequence stars. Given this inconsistency, we can only conclude that the star should be rotating more slowly than a period of 22 days.

Stellar rotation can also be studied by analyzing the periodicity of the variation of the $\mathrm{Ca}$ II HK chromospheric activity index. Active regions coming in and out of view will induce a semiperiodic signal in the activity index and the RVs (Dumusque et al. 2011a). When looking at a generalized Lomb-Scargle periodogram (GLS; Zechmeister \& Kürster 2009) of the calcium activity index derived with HARPS-N spectra in Figure 1, signals with periods greater than 30 days can be found. However these signals all have a false alarm probability (FAP) greater than $10 \%$ implying that there is no significant variation in the activity level, which is generally the case for slow rotators that exhibit a low activity level.

\subsubsection{Kepler-10 Rotational Period Estimation with Projected Rotational Velocity}

Another way of estimating the rotational period of the star is using the projected rotational velocity $v \sin i$ measured on the stellar spectra, as rotation will broaden spectral lines. As we can see in Table 2 , the value of $0.6 \mathrm{~km} \mathrm{~s}^{-1}$ derived by fitting HARPS-N spectra with synthetic spectra is extremely low (using the SPC code). This value is consistent with the one derived in the discovery paper using the SME code (Valenti \& Piskunov 1996). These techniques are not sensitive below $\sim 2 \mathrm{~km} \mathrm{~s}^{-1}$ because of the limited instrumental resolution. Another way of estimating the $v \sin i$ is to look at the FWHM of the cross correlation function used to derive the RVs (Boisse et al. 2010; Santos et al. 2002). In this case, the instrumental FWHM is $5.9 \pm 0.2 \mathrm{~km} \mathrm{~s}^{-1}$ at $(B-V)=0.7$, and Kepler-10 has a FWHM of $6.41 \mathrm{~km} \mathrm{~s}^{-1}$. Using the numerical factor of 1.23 from Hirano et al. (2010) valid for slow rotators, we arrive to $v \sin i=\sqrt{\mathrm{FWHM}^{2}-\mathrm{FWHM}_{\mathrm{inst}}{ }^{2}} / 1.23=2.04 \pm 0.34 \mathrm{~km} \mathrm{~s}^{-1}$. Assuming that the star is seen equator on, which is probable given that we have a multi-planet transiting system (Hirano et al. 2014), and assuming a radius of $1.065 R_{\odot}$, our two $v \sin i$ estimates point to slow rotational periods of 90 and 26 days, respectively.

\subsubsection{Kepler-10 Rotational Period Estimation Using the Kepler Photometry}

Finally, rotation can also be studied with Kepler light curves by searching for flux variations induced by active regions coming in and out of view. As these active regions rotate with the star, a signal with a period similar to the rotation of the star is expected. Depending on the coverage and evolution of active regions, the amplitude of the activity signal can strongly vary from one rotational period to the next because these regions only live for a short amount of time. On the Sun, the lifetime of active regions is about a few rotational periods (e.g., Howard 2000). 

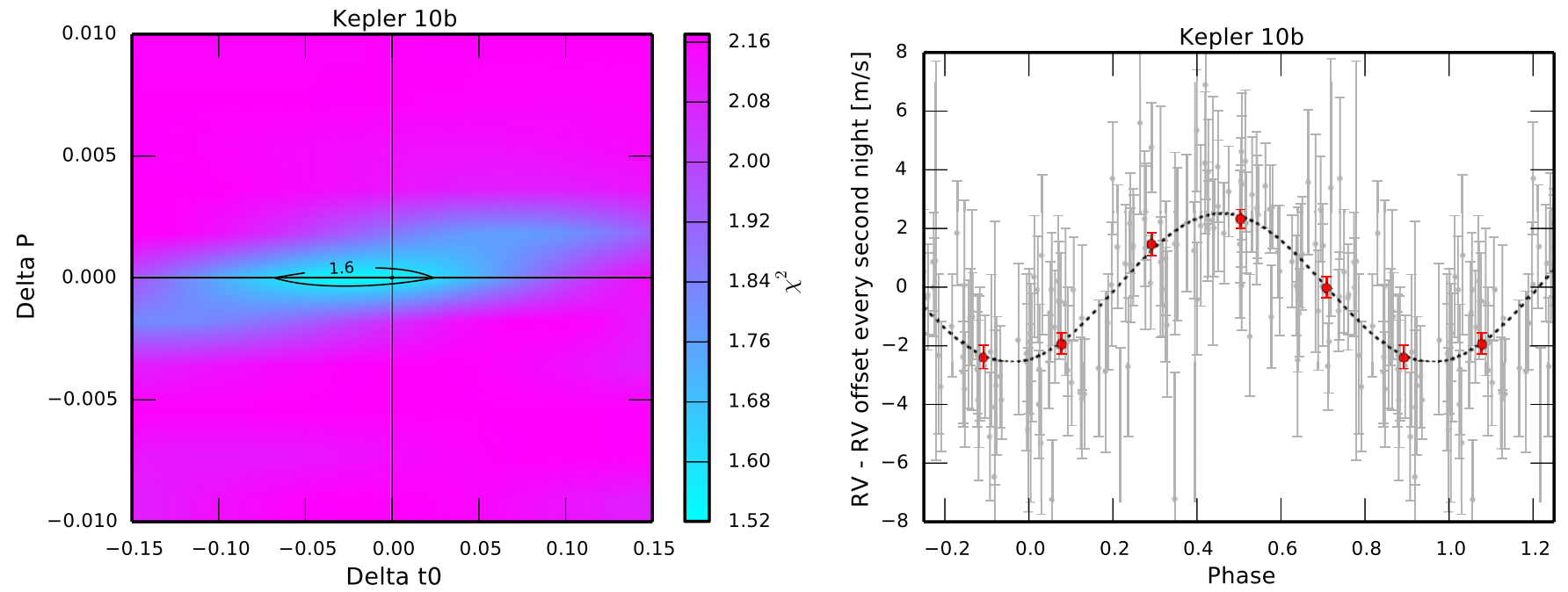

Figure 2. Radial velocity fit of Kepler-10b using a circular orbit model plus RV offsets every two nights. Left: contour plot of the $\chi^{2}$ surface around the ephemeris for the period and epoch of transit given by the published Kepler photometric solution (origin of the axis). The minimum $\chi^{2}$ fit obtained with the RV measurements matches the photometric solution. Right: phase-folded radial velocity signal of Kepler-10b. The gray points corresponds to all the RV measurements obtained for Kepler-10, the big red dots represent the same data binned in phase with a window of 0.2 , and the black dotted line our best fit for Kepler-10b.

(A color version of this figure is available in the online journal.)

Kepler light curves over time spans of dozens of days are strongly affected by instrumental systematics. As clearly stated in the Kepler Data Release 21 Notes, the detrended light curves (PDCSAP) should not be used to look for stellar rotational periods greater than 20 days, as the pipeline removes all signals on longer timescales. Because all the rotation indicators discussed in the preceding sections agree on a rotational period greater than 20 days, we did not attempt to derive the rotational period of Kepler-10 using the Kepler photometry.

We used different indicators to infer the rotational period of Kepler-10. None of these indicators can give us a precise value, but they all agree that the star rotates with a period longer than 20 days.

\subsection{Kepler-10b}

Kepler-10b has an orbital period of 0.84 days, and therefore several measurements per night were obtained to sample efficiently the planetary signal. The other signal expected in the RVs of Kepler-10 is the one coming from Kepler-10c with a 45 day period, if detectable. Considering these signals, the RV variation expected over two days is dominated by Kepler-10b, and as was done for Kepler-78b (Pepe et al. 2013), we decided to adjust the RV offset every two nights to filter out the signal of Kepler-10c (Hatzes et al. 2010). Assuming that the eccentricity of Kepler-10b is small (Fogtmann-Schulz et al. 2014), we fit the RVs with a model composed of a circular orbit plus an RV offset for every two nights. Repeating this optimization over a grid of orbital frequencies and times of mid-transit, we confirm that the RV signal of Kepler-10b is consistent with the photometric transit ephemeris (see Figure 2, left).

The best fit for the planetary signal, with a reduced $\chi^{2}$ of 1.52 , converges to a semi-amplitude of $K=2.51 \pm 0.32 \mathrm{~m} \mathrm{~s}^{-1}$. This semi-amplitude, although smaller, is compatible with the discovery solution, $K=3.3_{-1}^{+0.8} \mathrm{~m} \mathrm{~s}^{-1}$. The phase-folded RV signal of Kepler-10b, with the RV offset corrected every two nights can be found in Figure 2, right.

\subsection{Kepler-10c}

In the preceding section, we analyzed the data without considering Kepler-10c. The signal of this planet, if detectable, was filtered out by adjusting the RV offset every two nights. In this section, we do not utilize this filtering and instead search for significant signals in the GLS (Zechmeister \& Kürster 2009) of the raw RVs.

When looking at the GLS periodogram (see Figure 3, left), it is clear that a signal at 45 days emerges from the noise, even when the signal of Kepler-10b is not removed. To push the analysis further and check that this 45-day signal corresponds to Kepler-10c and not stellar activity, we removed the signal of Kepler-10b by fitting a circular orbit, fixing the period and the transit time to the published values (Batalha et al. 2011), and leaving the amplitude as a free parameter. We then compared the GLS periodogram of the RV residuals for the measurements obtained in 2012, the ones obtained in 2013, and for the entire data set. As we can see in Figure 3, right, the 45-day signal can be seen in each individual subset. The period of this signal is not well constrained, but it has the same phase from one subset to the other, which is expected for a planetary signal. The semi-amplitudes found, fitting a circular orbit with the published photometric ephemeris of Kepler-10c (Fressin et al. 2011 ), are $3.38 \pm 0.62,3.74 \pm 0.44$, and $3.11 \pm 0.35 \mathrm{~m} \mathrm{~s}^{-1}$ for the measurements obtained in 2012, the ones obtained in 2013, and for the entire data set, respectively. The good agreement between these amplitudes, in addition to the fact that the signal keeps the same phase from one year to the next, are strong arguments in favor of the planetary nature of the signal.

\subsection{MCMC Analysis of the RVs Assuming Gaussian Noise with Priors from Kepler Photometry}

The preceding section showed that the signal of Kepler-10c is present in the RV data. The next step is to obtain reliable parameters for the mass of this planet. ${ }^{18}$ The Kepler photometry exhibits 1124 transits of Kepler-10b and 24 transits of Kepler$10 \mathrm{c}$, which gives us a very-high precision on the period and the transit epoch for both planets. The RV measurements sample $\sim 350$ periods of Kepler-10b and $\sim 7$ periods of Kepler-10c, with a much sparser sampling. From those numbers, it is clear that

18 The orbital inclination of Kepler-10c is 89.7 (Fressin et al. 2011), therefore the minimum mass of the planet is equal to the real mass. 

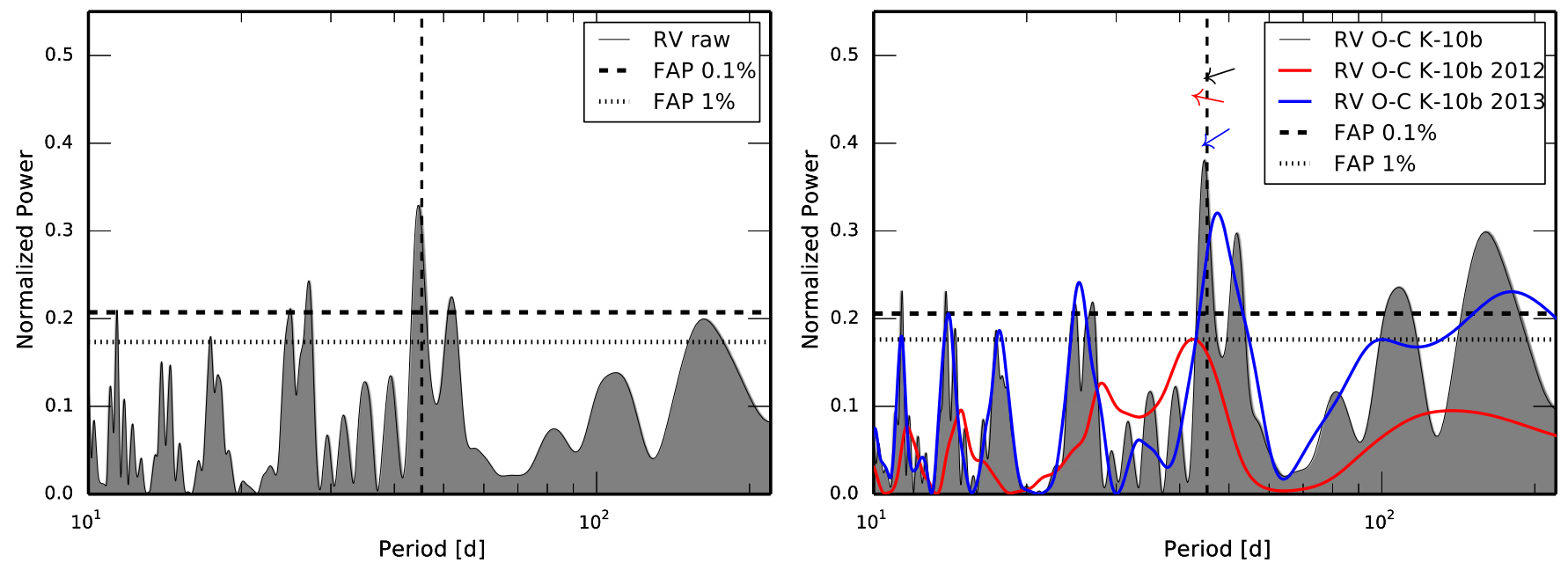

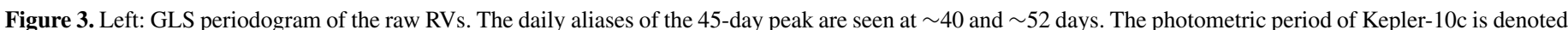

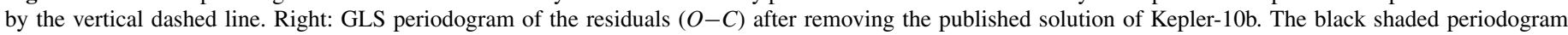

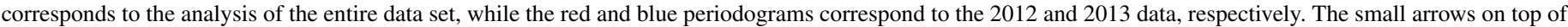

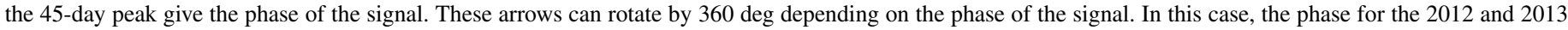

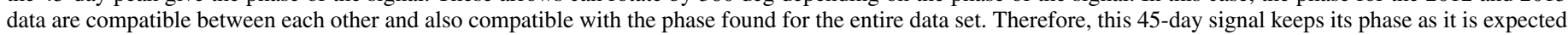
for a planet. For each plot, the horizontal lines correspond, from top to bottom, to a FAP level of $0.1 \%$ and $1 \%$.

(A color version of this figure is available in the online journal.)

the period and the transit time are constrained by the photometry itself and that RV measurements will not help to improve the determination of these parameters. We therefore decided to fit the RV data only, using the strong priors on the planetary periods and times of transit given by the photometry. These parameters were estimated by fitting, on all the available Kepler shortcadence simple-aperture-photometry data ${ }^{19}$ (up to quarter Q17, with a $5 \sigma$ outlier rejection), a model for each transit (Giménez 2006), and a straight line to the individual transit times. The ephemeris values are reported in Table 3 and are consistent within $1 \sigma$ with those previously derived by Batalha et al. (2011) and later on by Fogtmann-Schulz et al. (2014).

The model that is fitted to the RV data includes two Keplerian signals, one for each planet, plus a RV offset $R V_{0}$ to account for a possible offset between the data taken with the old and the new HARPS-N CCD:

$$
\begin{aligned}
\Delta \mathrm{RV}\left(t_{i}\right)= & \gamma+\mathrm{RV}_{0}\left(t_{i}\right)+\sum_{j=1}^{j=2} K_{j}\left[\cos \left(\theta\left(t_{i}, T_{0, j}, P_{j}, e_{j}\right)+\omega_{j}\right)\right. \\
& \left.+e_{j} \cos \left(\omega_{j}\right)\right] .
\end{aligned}
$$

In this formula, $\gamma$ is the systemic velocity of Kepler-10, and $\mathrm{RV}_{0}\left(t_{i}\right)=0$ for $t_{i}<\mathrm{BJD}=2456185$ and a constant for $t_{i}>$ $\mathrm{BJD}=2456185$. Each planet $j$ is characterized by its semiamplitude $K_{j}$, period $P_{j}$, time of periastron $T_{0, j}$, eccentricity $e_{j}$ and argument of periastron $\omega_{j}$. The function $\theta\left(t_{i}, T_{0, j}, P_{j}, e_{j}\right)$ is the true anomaly of the planet at time $t_{i}$. In addition to this model, we also consider that Gaussian noise of stellar origin could affect the RVs. The choice of a Gaussian noise term was made because Kepler-10 does not show any significant sign of activity. Therefore, correlation between measurements due to active regions drifting on the stellar surface is not expected. However, granulation phenomena induced by stellar convection are known to induce correlated noise in the RVs (Dumusque et al. 2011b). With an effective temperature similar to the Sun, super granulation, which is the most important phenomenon

\footnotetext{
19 http://keplergo.arc.nasa.gov/PyKEprimerLCs.shtml (Jenkins et al. 2010)
}

of granulation, should induce a RV rms smaller than $1 \mathrm{~m} \mathrm{~s}^{-1}$ on the timescale of a day. This should perturb the RV signal of Kepler-10b only slightly because the RV semi-amplitude of this planet is three times bigger. In addition, the strategy of observing the target twice per night, over more than 70 nights, allows us to average out this correlated noise. Regarding Kepler$10 \mathrm{c}$, we do not expect any perturbation as the timescale of super granulation and the planetary period are totally different. We concluded that no significant correlated noise should affect the RV measurements of Kepler-10, and we decided to only consider Gaussian noise when fitting the data.

Before starting any fitting procedure on the data, the $T_{0, j}$ values for both planets were shifted close to the average date of the HARPS-N observations to limit error propagation when fitting the periods $P_{j}$ and epochs $T_{0, j}$. In addition, we used $C_{j}=\sqrt{e_{j}} \cos \left(\omega_{j}\right)$ and $S_{j}=\sqrt{e_{j}} \sin \left(\omega_{j}\right)$ as free parameters of the fit instead of $e_{j}$ and $\omega_{j}$. This modification allows a more efficient exploration of the parameter space in the case of small eccentricities (Ford 2006), which is the case for Kepler-10b and c (Fogtmann-Schulz et al. 2014). The model is then fitted to the data using a Markov Chain Monte Carlo (MCMC) algorithm that is similar to the one recently applied to the CoRoT-7 system (see R. D. Haywood et al., in preparation). The stellar signal contribution is modeled as a constant jitter term $\sigma_{s j}$ in addition to the RV instrumental noise $\sigma_{i}$ returned by the HARPS-N pipeline. The following likelihood:

$$
\mathcal{L}=\frac{1}{\sqrt{2 \pi\left(\sigma_{i}^{2}+\sigma_{s j}^{2}\right)}} \exp \left[-\frac{\left(\mathrm{RV}\left(t_{i}\right)-\Delta_{\mathrm{RV}}\left(t_{i}\right)\right)^{2}}{2\left(\sigma_{i}^{2}+\sigma_{s j}^{2}\right)}\right]
$$

is used for the MCMC. Uniform priors were set on all the parameters with the constrain that the RV jitter and semiamplitudes of both Kepler-10b and c must be greater or equal than zero. Gaussian priors for the period and the transit epoch of both planets were imposed based on our previous photometric ephemeris determination (see Table 3).

Although Fogtmann-Schulz et al. (2014) find very low eccentricities for both planets, we decided to test a model with free 
Table 3

Kepler-10 Planetary System Parameters

\begin{tabular}{lcr}
\hline \hline Stellar Parameters & RV Only & RV and Photometry \\
\hline Limb-darkening coefficient $u_{a}$ & $\ldots$ & $0.40 \pm 0.07$ \\
Limb-darkening coefficient $u_{b}$ & $\ldots$ & $0.27 \pm 0.10$ \\
Systemic velocity $\gamma\left(\mathrm{m} \mathrm{s}^{-1}\right)$ & $-98739.90 \pm 0.42$ & $-98739.89 \pm 0.43$ \\
RV offset $\mathrm{RV}_{0}\left(\mathrm{~m} \mathrm{~s}^{-1}\right)$ & $1.83 \pm 0.45$ & $1.78 \pm 0.32$ \\
Radial-velocity jitter $\left(\mathrm{m} \mathrm{s}^{-1}\right)$ & $2.45_{-0.21}^{+0.23}$ & $2.47 \pm 0.23$ \\
\hline
\end{tabular}

\begin{tabular}{|c|c|c|}
\hline \multicolumn{3}{|c|}{ Kepler-10b } \\
\hline Transit and orbital parameters & \\
\hline Orbital period $P$ (days) & \multicolumn{2}{|c|}{$0.8374907 \pm 0.0000002$} \\
\hline Transit epoch $T_{0}\left(\mathrm{BJD}_{\mathrm{TDB}}-2454900\right)$ & \multicolumn{2}{|c|}{$134.08687 \pm 0.00018$} \\
\hline Transit duration $T_{14}(\mathrm{hr})$ & $\ldots$ & $1.8110_{-0.0036}^{+0.0064}$ \\
\hline Radius ratio $R_{\mathrm{p}} / R_{*}$ & $\ldots$ & $0.01261_{-0.00013}^{+0.00026}$ \\
\hline Inclination $i(\mathrm{deg})$ & $\ldots$ & $84.8_{-3.9}^{+3.2}$ \\
\hline$a / R_{*}$ & $\ldots$ & $3.46_{-0.28}^{+0.14}$ \\
\hline Impact parameter $b$ & $\ldots$ & $0.31 \pm 0.19$ \\
\hline Orbital eccentricity $e$ & \multicolumn{2}{|c|}{0 (fixed) } \\
\hline Radial-velocity semi-amplitude $K\left(\mathrm{~m} \mathrm{~s}^{-1}\right)$ & $2.38 \pm 0.34$ & $2.38 \pm 0.35$ \\
\hline \multicolumn{3}{|l|}{ Planetary parameters } \\
\hline Planet mass $M_{\mathrm{p}}\left(M_{\oplus}\right)$ & $\ldots$ & $3.33 \pm 0.49$ \\
\hline Planet radius $R_{\mathrm{p}}\left(R_{\oplus}\right)$ & $\ldots$ & $1.47_{-0.02}^{+0.03}$ \\
\hline Planet density $\rho_{\mathrm{p}}\left(\mathrm{g} \mathrm{cm}^{-3}\right)$ & $\ldots$ & $5.8 \pm 0.8$ \\
\hline Planet surface gravity $\log g_{\mathrm{p}}(\mathrm{cgs})$ & $\ldots$ & $3.18_{-0.07}^{+0.06}$ \\
\hline Orbital semi-major axis $a(\mathrm{AU})$ & $\ldots$ & $0.01685 \pm 0.00013$ \\
\hline Equilibrium temperature $T_{\mathrm{eq}}(\mathrm{K})$ & $\ldots$ & $2169_{-44}^{+96}{ }^{a}$ \\
\hline
\end{tabular}

Transit and orbital parameters

Kepler-10c

Orbital period $P$ [days)

Transit epoch $T_{0}\left(\mathrm{BJD}_{\mathrm{TDB}}-2454900\right)$

Transit duration $T_{14}(\mathrm{hr})$

Radius ratio $R_{\mathrm{p}} / R_{*}$

Inclination $i(\mathrm{deg})$

$a / R_{*}$

Impact parameter $b$

Orbital eccentricity $e$

Radial velocity semi-amplitude $K\left(\mathrm{~m} \mathrm{~s}^{-1}\right)$

$45.294301 \pm 0.000048$

$162.26648 \pm 0.00081$

.. $6.898_{-0.023}^{+0.058}$

$\begin{array}{ll}\cdots & 0.02019_{-0.00025}^{+0.00001}\end{array}$

$\quad 89.59_{-0.43}^{+0.25}$

$47.9_{-7.2}^{+2.8}$

$0.36_{-0.20}^{+0.25}$

Planetary parameters

Planet mass $M_{\mathrm{p}}\left(M_{\oplus}\right)$

Planet radius $R_{\mathrm{p}}\left(R_{\oplus}\right)$

Planet density $\rho_{\mathrm{p}}\left(\mathrm{g} \mathrm{cm}^{-3}\right)$

Planet surface gravity $\log g_{\mathrm{p}}(\mathrm{cgs})$

Orbital semi-major axis $a$ (AU)

Equilibrium temperature $T_{\text {eq }}(\mathrm{K})$
$3.25 \pm 0.36 \quad 3.26 \pm 0.36$

$\begin{array}{lc}\ldots & 17.2 \pm 1.9 \\ \cdots & 2.35_{-0.04}^{+0.09} \\ \cdots & 7.1 \pm 1.0 \\ \cdots & 3.48_{-0.06}^{+0.05} \\ \cdots & 0.2410 \pm 0.0019 \\ \cdots & 584_{-17}^{+50} \mathrm{a}\end{array}$

Notes. Kepler-10 planetary system parameters for the circular solution using the RV data only (with priors from photometry), and for the circular solution with the combined photometric and RV analysis. The error bars represent a $1 \sigma$ uncertainty on the parameters.

${ }^{a}$ Black body equilibrium temperature assuming a zero Bond albedo and uniform heat redistribution to the night-side (Rowe et al. 2006).

orbital eccentricities (13 parameters) as well as one with eccentricities fixed to zero ( 9 parameters). In order to assess which model is best, we calculated the marginal likelihood of each model according to the method of Chib \& Jeliazkov (2001) (see the Appendix in R. D. Haywood et al., in preparation), which uses the posterior distributions of the MCMC chain. We obtained a Bayes factor of 10 in favor of the model with fixed zero eccentricities. While this does not constitute strong evidence, according to Jeffreys (1961), in favor of circular orbits, it implies that the penalty induced by the extra parameters needed to allow free eccentricities outweighs the increase in likelihood brought by the improvements to the fit. Indeed, when the eccentricities are free to vary, we obtain $0<e_{b}<0.12$ and $0<e_{c}<0.14$ which suggests that both orbits are compatible with circular orbits. The outcome of the circular orbit fit is shown in Table 3, where the modes of the marginal posteriors with their errors are shown. The phase-folded RVs with the best fit model for Kepler-10b and c can be found in Figure 4, and the posteriors for all the parameters with their mutual correlation can be found in Figure 5.

The RV semi-amplitudes found for Kepler-10b and c are in agreement with our preceding analyses within $1 \sigma$ (see 

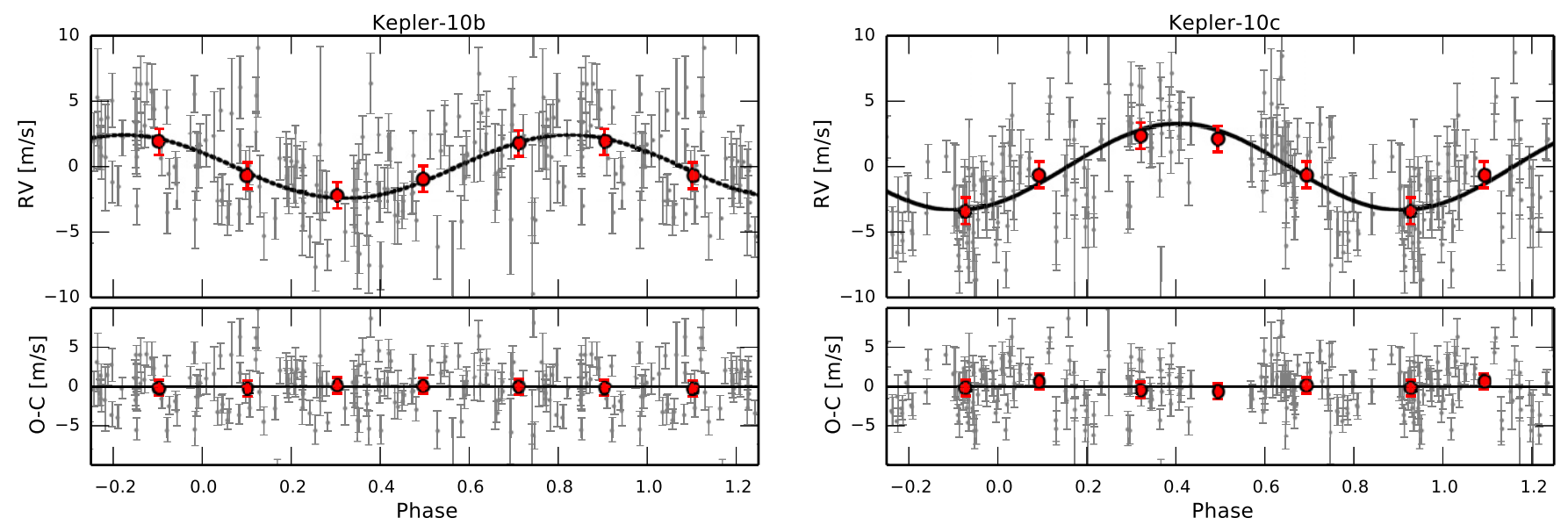

Figure 4. Best phase-folded solution for the fit of Kepler-10b and $\mathrm{c}$ on the RVs only, assuming circular orbits and an additional constant stellar jitter. The gray points corresponds to all the RV measurements obtained for Kepler-10, the big red dots represent the same data binned in phase with a window of 0.2 , and the black dotted lines our best fit for Kepler-10b and c.

(A color version of this figure is available in the online journal.)

Sections 4.3 and 4.4). These semi-amplitudes are estimated with an uncertainty of $14 \%$ and $11 \%$, respectively. Looking at the GLS periodogram of the RV residuals after removing our best fit solution (see the left plot of Figure 6), the highest signal remaining in the data is found at $\sim 17$ days, with a FAP of $0.5 \%$. The FAP of this signal is significant enough to try a three-planet model fit. Performing an MCMC with three circular planets converges to a realistic solution. Calculating the Bayes factor between the two and three-planet models results in a highly significant factor of $e^{14}$ in favor of the more complex model. However, looking at the periodogram for each season of observation (see the right plot of Figure 3), the 17-day signal is only present in the second season. This signal is thus not a coherent signal of constant amplitude spanning both seasons, and therefore cannot be associated to a planet. When removing the $\mathrm{RV}$ measurements between $\mathrm{BJD}=2456350$ and $\mathrm{BJD}=2456450$ ( 31 points in total, equal to $20 \%$ of all the measurements), the 17-day signal disappear from the residuals (see the right plot of Figure 6). These rejected RV measurements seem affected by small activity variations that can be seen when looking at the calcium activity index variation (see left plot of Figure 1). The periodogram of the calcium activity index (see right plot of Figure 1) shows important, however, not significant signals at 34 days and at the first harmonic, i.e., 17 days. It is thus possible that the 17-day signal present in the RVs residuals is the first harmonic of the stellar rotation. This 17-day period signal, highly significant when estimating Bayes factor assuming an extra planet, seems to be instead due to short-term activity variations. Even though Bayes factor favors a more complex model including an extra planet, an in-depth analysis is required to characterize the real nature of this extra signal.

\subsection{Combined MCMC Analysis of the RVs and Kepler Photometry Considering RV Gaussian Noise}

In this section, we proceed to a combined RV and photometric fit to determine the orbital and physical parameters of Kepler$10 \mathrm{~b}$ and $\mathrm{c}$ by taking advantage of all the available Kepler data.

To derive the Kepler-10 system parameters, a Bayesian combined analysis of the Kepler short-cadence photometry and HARPS-N RV measurements was performed using a Differential Evolution Markov Chain Monte Carlo method (DE-
MCMC; Eastman et al. 2013; Ter Braak 2006). For this purpose, the epochs of the HARPS-N observations were converted from BJD $_{\text {UTC }}$ into BJD $\mathrm{BDB}_{\mathrm{TDB}}$ (Eastman et al. 2010), which is the time stamp of Kepler data. The light curves of Kepler-10b and 10c were phase-folded with our new ephemeris (see Table 3 and discussion in Section 4.5) and binned in samples of $4 \mathrm{~s}$ and $30 \mathrm{~s}$, respectively, to significantly reduce the computation time of our combined analysis.

The HARPS-N RVs and the phase-folded and binned light curves of Kepler-10b and c were simultaneously fitted by considering a two-planet model with two Keplerian orbits and two transit models (Giménez 2006). Circular orbits were adopted for both planets based on the strong constraints from the asteroseismic density determination (Fogtmann-Schulz et al. 2014) and our analysis of HARPS-N RV measurements in Section 4.5. In total, our model has seventeen free parameters: the transit epochs, orbital periods, RV semi-amplitudes, transit durations, orbital inclinations, and planet-to-stellar radius ratios of Kepler-10b and c, the stellar systemic velocity, a RV offset between the HARPS-N measurements obtained with the old and the new CCD, an uncorrelated RV jitter term (e.g., Gregory 2005), and the limb-darkening coefficients which were fitted by using the triangular sampling method as suggested by Kipping (2013): $q_{1}=\left(u_{a}+u_{b}\right)^{2}$ and $q_{2}=0.5 u_{a} /\left(u_{a}+u_{b}\right)$, where $u_{\mathrm{a}}$ and $u_{\mathrm{b}}$ are the linear and quadratic coefficients of the limb-darkening quadratic law.

A Gaussian likelihood was maximized in our Bayesian analysis (see Equation (2)). Thirty-four DE-MCMC chains, i.e., twice the number of free parameters, were run simultaneously, after being started at different positions in the parameter space but reasonably close to the system values. The jumps of a given chain in the parameter space were determined from two random chains, according to the prescriptions given by Ter Braak (2006). The Metropolis-Hastings algorithm was used to accept or reject a proposal step for each chain. Uniform priors were set on all parameters with the constraints that the RV jitter and semiamplitudes of both Kepler-10b and 10c must be greater or equal than zero, and $q_{1}$ and $q_{2}$ are allowed to vary in the interval $[0,1]$ (Kipping 2013). Gaussian priors were imposed to the transit epochs and orbital periods based on our previous ephemeris determination (see Table 3). Unlike Fogtmann-Schulz et al. (2014), no prior was set on $a / R_{\star}$ because this prior strongly 

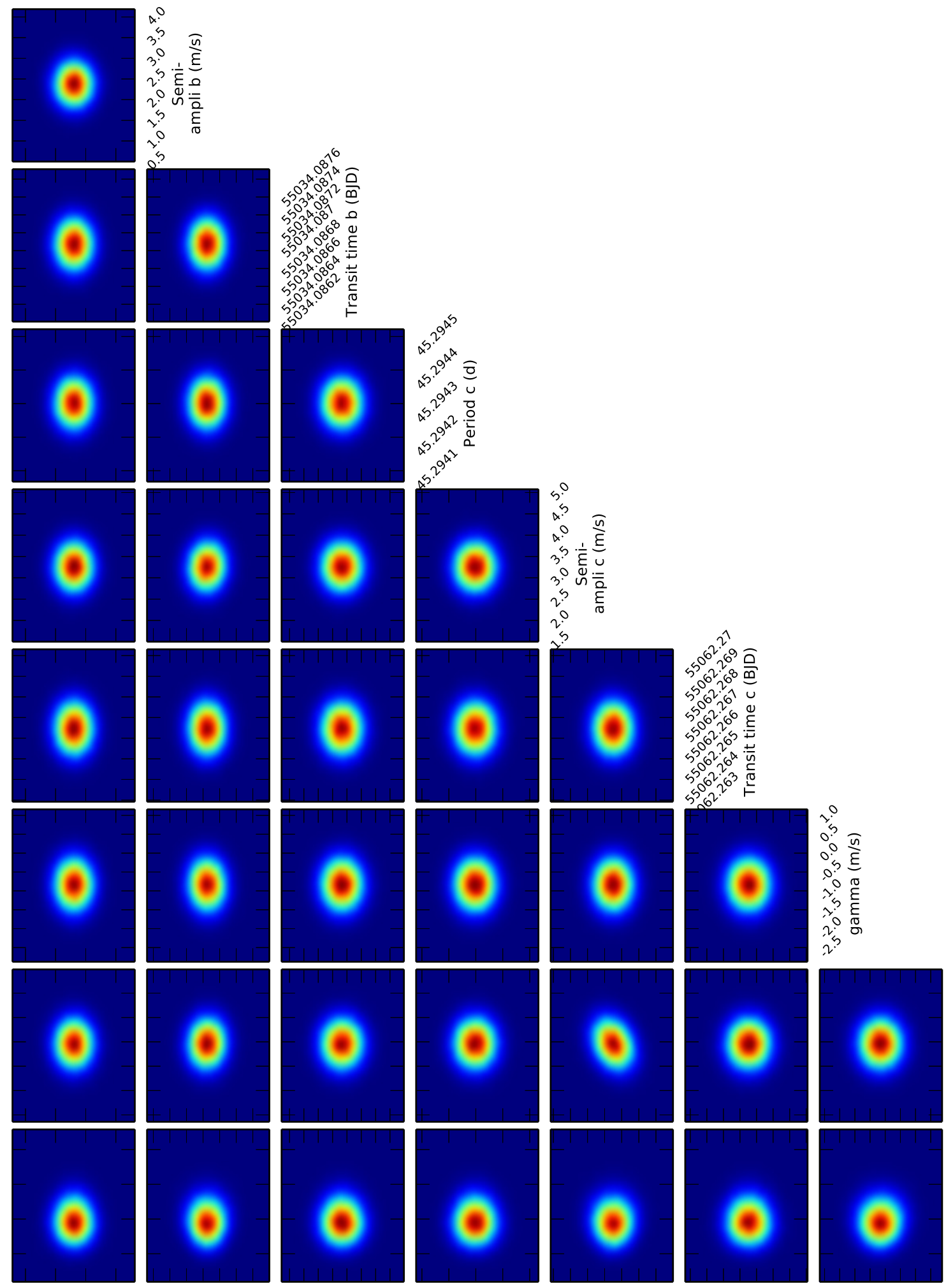

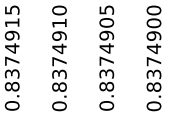

(p) $q$ po!lı⿰丿⿱丄𠃍

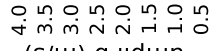
(s/u) $q$ !ldure

$$
\text { -!üs }
$$

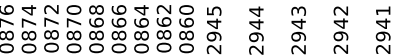

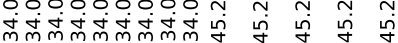

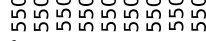
(alg) q әuा zाsued

\begin{abstract}
(p) ว po!ıәd
\end{abstract}
(s/u) 0 mdue

-!üs

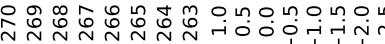

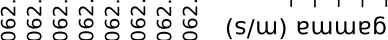

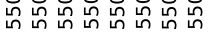

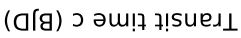

(s/u) ұәऽң० $\wedge$ y

Figure 5. Posterior distributions and correlation between the different MCMC parameters for the model with Gaussian noise and circular orbits. "RV offset" corresponds to the RV offset between the old and the new HARPS-N CCD, while "gamma" is the systemic velocity of Kepler- 10 .

(A color version of this figure is available in the online journal.) 

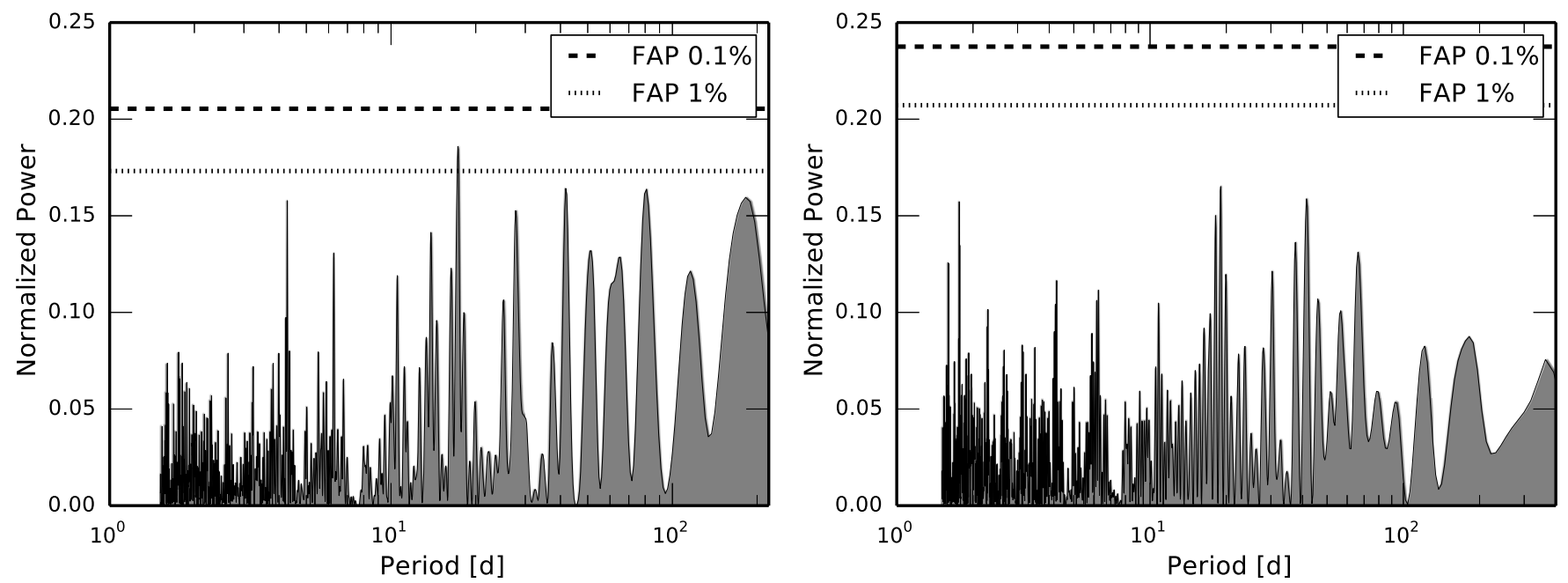

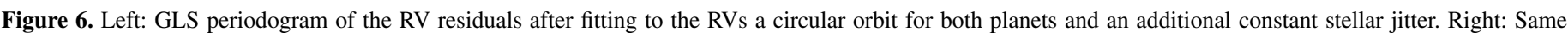

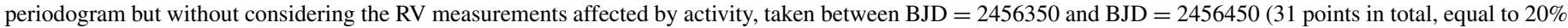

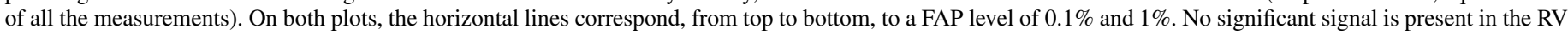
residuals.
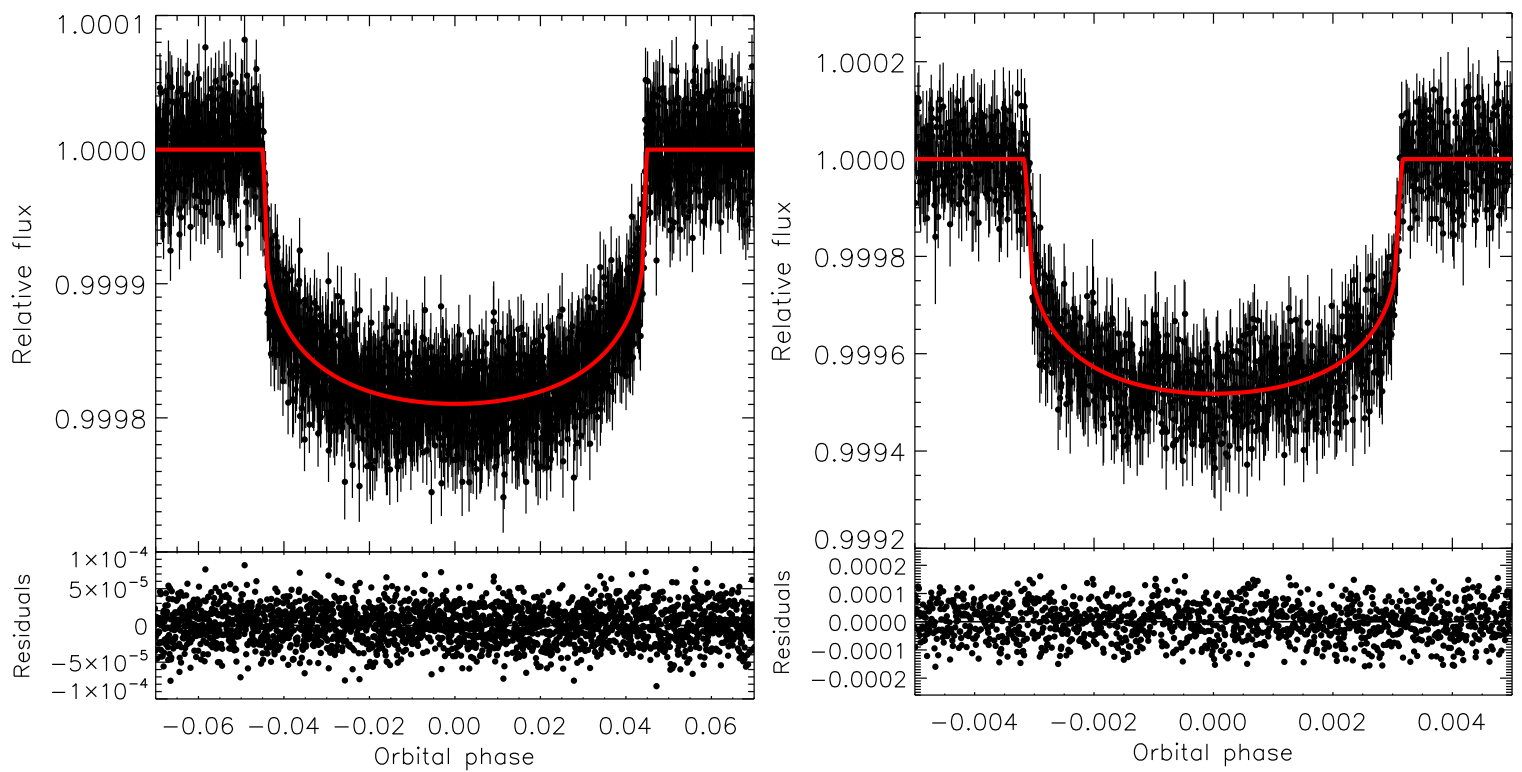

Figure 7. Phase-folded and binned transits of Kepler-10b (left) and c (right) along with the best-fit models.

(A color version of this figure is available in the online journal.)

affects the posterior distributions of transit parameters and yields unrealistic error bars.

The DE-MCMC analysis was stopped after convergence and well mixing of the chains were reached according to the Gelman-Rubin statistics ( $\hat{R}<1.03$ for all parameters; Gelman et al. 2004). Steps belonging to the "burn-in" phase were identified following Knutson et al. (2009) and excluded. Figure 7 shows the phase-folded and binned transits of Kepler-10b and $\mathrm{c}$ along with the best-fit models. The linear and quadratic limbdarkening coefficients $u_{a}=0.40 \pm 0.07$ and $u_{b}=0.27 \pm 0.10$ agree well with values predicted by Kurucz stellar models for the Kepler bandpass (Sing 2010), i.e., $u_{a}=0.390 \pm 0.006$ and $u_{b}=0.264 \pm 0.004$. We derived a radius of $1.47_{-0.02}^{+0.03} R_{\oplus}$, a mass of $3.33 \pm 0.49 M_{\oplus}$, and a density of $5.8 \pm 0.8 \mathrm{~g} \mathrm{~cm}^{-3}$ for Kepler$10 \mathrm{~b}$ and a radius of $2.35_{-0.04}^{+0.09} R_{\oplus}$, a mass of $17.2 \pm 1.9 M_{\oplus}$, and a density of $7.1 \pm 1.0 \mathrm{~g} \mathrm{~cm}^{-3}$ for Kepler-10c.

\section{KEPLER-10C DENSITY}

Understanding the transition from rocky planets to planets defined by a hydrogen-dominated envelope is critical to planet formation theory. Kepler-10c stands out in the mass-radius diagram (Figure 8), in direct challenge to theory.

To begin with, it is possible to show that Kepler-10c cannot possess a hydrogen-dominated envelope. At $0.24 \mathrm{AU}$ from a solar-luminosity star of very old age, Kepler-10c is too hot $\left(T_{\mathrm{eq}}=584 \mathrm{~K}\right)$ to retain an outgassed hydrogen atmosphere, or an accreted hydrogen-helium mixture, as shown by applying Rogers et al. (2011) calculations for $T_{\mathrm{eq}}=500 \mathrm{~K}$ and the radius of Kepler-10c (all loss timescales are shorter than $1 \mathrm{Gyr}$ ). Had Kepler-10c accreted a massive hydrogen-helium envelope 10.6 Gyr ago and still kept some of it, its radius would have to be larger than $3 R_{\oplus}$, similar to Kepler-20c (at 15.7 $M_{\oplus}$ in Figure 8). Therein lies the challenge, as Kepler-10c is significantly above 


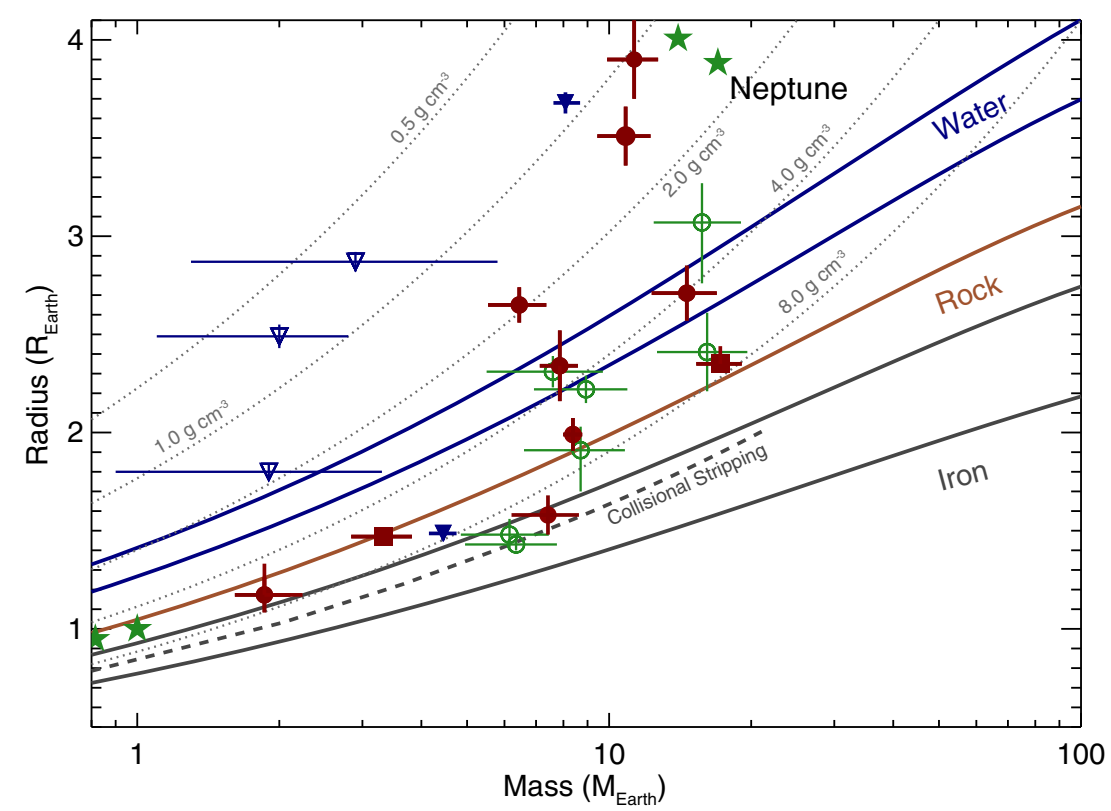

Figure 8. Mass-radius diagram for planets with radius smaller than $4 R_{\oplus}$ and that present a mass determination better than $30 \%$. The only exception are the planets from the Kepler-11 planetary system that we wanted to show here because they have raised the issue that there may be planets with extended envelopes of $\mathrm{H}$ and $\mathrm{He}$ even at masses less than $5 M_{\oplus}$. Planets with mass determined by the RV technique are represented by circles (except squares to highlight Kepler-10b and c). Planets with mass determined by TTVs are represented by triangles. Filled symbols are used when the precision on the mass is smaller than $20 \%$, highlighting measurements where an in-depth analysis of the planet composition can be done, while open symbol are used when the precision on the mass is higher than $20 \%$. The dashed curve is the maximum collisional stripping curve by Marcus et al. (2010), which gives the minimum radii of super-Earths assuming giant impacts during formation. Kepler-10c is the only planet more massive than $10 M_{\oplus}$ with such a high density, for which the precision in mass is better than $20 \%$.

(A color version of this figure is available in the online journal.)

the theoretical critical mass of 10-12 $M_{\oplus}$ enabling envelope accretion (Ikoma \& Hori 2012; Lopez \& Fortney 2013), but has none.

Kepler-10c is best characterized as a solid planet, implying that its bulk composition is dominated by rocks (silicates, judging from the star's composition) and a significant amount of volatile material of high mean molecular weight (i.e., water) of 5 to $20 \mathrm{wt} . \%$. Even if that water is in a separate envelope, which is likely if it is more than 10 to $15 \mathrm{wt} . \%$ (Elkins-Tanton 2011), most of it will be in the form of solid high-pressure ices at age 10.6 Gyr (Zeng \& Sasselov 2014). The precise amount of water in Kepler-10c cannot be constrained further, given the known compositional degeneracy of the mass-radius diagram (Valencia et al. 2007).

We note in Figure 8 that Kepler-10c is not alone in this region of the mass-radius diagram, as Kepler-131b $\left(P_{\text {orb }}=16\right.$ days, $16.1 \pm 3.5 M_{\oplus}, 2.4 \pm 0.2 R_{\oplus}$; Marcy et al. 2014) lies very close to it. However, the mass determination of Kepler-131b is not as robust as for Kepler-10c and more data are required to asses if this planet is a twin of Kepler-10c.

Regardless of any water, Kepler-10c is a clear outlier, being many sigmas smaller in size than the densest Neptune-mass exoplanet Kepler-20c. Except Kepler-131b for which the density still have to be measured more precisely, the exoplanets that appear closest to Kepler-10c in composition, $55 \mathrm{Cnc}$ e and Kepler-20b, are half its mass. They are also both extremely close to their stars and hot, so it is not entirely clear how much the water they contain would contribute to their radii.

Kepler-10 has a space motion and an age characteristic of the old thick disk population, though with $[\mathrm{Fe} / \mathrm{H}]=-0.15$, it is not as metal poor as the typical thick disk metallicity of about $[\mathrm{Fe} / \mathrm{H}]=-0.6$. This implies caution regarding the $\mathrm{Fe} / \mathrm{Mg}$ and $\mathrm{Fe} / \mathrm{Si}$ ratios in the proto-planetary disk from which Kepler-10b and 10c formed. The model composition curves on Figure 8 assume solar ratios (Zeng \& Sasselov 2013); the expected variations due to expected enhancements are of $1 \%-2 \%$ in planet radius (Grasset et al. 2009). Kepler-10b and c might differ in their internal structure in the amount of $\mathrm{Fe}$ that is differentiated in their core; for the high mass of Kepler-10c full differentiation is questionable, but given its water content, the oxidation state of Fe is unclear (Zeng \& Sasselov 2013). These uncertainties and the comparison to Kepler-10b imply that Kepler-10c could also have no bulk water, which remains the best composition for Kepler-10b.

\section{CONCLUSION}

We revisited the Kepler-10 planetary system using the HARPS-N spectrograph. In total, 148 high-quality RV measurements of the star were obtained, which is nearly four times as many as the Keck-HIRES RV campaign reported in the discovery paper (Batalha et al. 2011). Using an optimized sampling, we were able to recover the signal of both planets, with a precision on the mass ${ }^{20}$ of $15 \%$ and $11 \%$ for Kepler- $10 \mathrm{~b}$ and c, respectively.

Kepler-10b is a hot rocky world with a mass of $3.33 \pm 0.49 M_{\oplus}$ and a radius of $1.47_{-0.02}^{+0.03} R_{\oplus}$, which implies a bulk density of $5.8 \pm 0.8 \mathrm{~g} \mathrm{~cm}^{-3}$, slightly higher than the Earth, and an internal structure and composition very similar to Earth.

Kepler-10c is a Neptune mass planet with a density higher than the Earth. Our best estimate of its mass is $17.2 \pm 1.9 M_{\oplus}$ for a radius of only $2.35_{-0.04}^{+0.09} R_{\oplus}$, which yields a density of $7.1 \pm 1.0 \mathrm{~g} \mathrm{~cm}^{-3}$. With these properties, Kepler-10c is

\footnotetext{
${ }^{20}$ It is not surprising that the precision on the mass of Kepler- $10 \mathrm{~b}$ is two times better than the previous value (28\%; Batalha et al. 2011). With four times as many observations of similar precision (see Pepe et al. 2013; Howard et al. 2013), we expect an improvement of a factor of two, everything else being equal.
} 
best characterized as a solid planet, implying that its bulk composition is dominated by rocks and a significant amount of water, about 5 to $20 \mathrm{wt} . \%$.

Kepler-10c might be the first firm example of a population of solid planets with masses above $10 M_{\oplus}$. From a recent theory of gas accretion at short orbital periods, the critical core mass required to accrete the gas present in the protoplanetary disc onto the planet is $M_{\mathrm{cr}} \sim 2.6 M \oplus(\eta / 0.3)^{1 / 2}\left(P_{\mathrm{orb}} / 1 \text { day }\right)^{5 / 12}$, where $\eta=M_{\mathrm{atm}} / M_{\mathrm{cr}}$ is the fractional mass comprised by the atmosphere (Rafikov 2006). If this model is correct, it implies that the core mass limit for gas accretion should increase with orbital period. A recent study from Buchhave et al. (2014), analyzing hundreds of Kepler candidates, seems to agree with this theoretical prediction. With a period of 45.29 days, a radius of $2.35 R_{\oplus}$, and a density higher than Earth, Kepler$10 \mathrm{c}$ would be at the limit of the transition from terrestrial to gaseous planets observed by Buchhave et al. (2014). Kepler-10c might be the first object confirming that longer period terrestrial planets can be more massive than ones with shorter periods. We note that Kepler-131b $\left(P_{\text {orb }}=16\right.$ days, $16.1 \pm 3.5 M_{\oplus}$, $2.4 \pm 0.2 R_{\oplus}$; Marcy et al. 2014) lies in the same location of the mass-radius diagram as Kepler-10c. However the mass determination of Kepler-131b is not as robust as for Kepler$10 \mathrm{c}$ and more data are needed to confirm the high density of this planet. Measuring precisely the mass of several other longperiod Kepler candidates orbiting bright stars could test this speculation. This experiment has just been started as a new observational program on HARPS-N.

The mass determination of Kepler-10c with HARPS-N shows once more how ground-based RV spectrographs and photometric transit surveys such as Kepler can work together to efficiently measure the densities of small planets. Such observational studies are crucial to constrain theoretical models of internal structure and composition of small-radius planets.

Although Kepler-10c induces a gravitational effect on its host star three times greater than the instrumental precision of HARPS-N, determining its mass to a satisfactory degree of precision required an important RV follow-up campaign; this is because Kepler-10 is a faint star for high-resolution spectrographs and thus the errors are dominated by photon noise. It is essential that future transit searches focus on bright stars in order to allow high-quality RV follow-up needed to measure planet masses with a $10 \%$ precision. The upcoming Kepler K2 mission (Howell et al. 2014) should provide soon a few interesting candidates. Then, in a few years, the TESS satellite (Ricker et al. 2010) should provide hundreds of candidates by performing an all sky survey of short-period transiting planets orbiting stars brighter than $V=12$. On the longer term, PLATO (Rauer et al. 2013) will look for habitable Earth-like planets around those same bright stars.

The HARPS-N project was funded by the Prodex Program of the Swiss Space Office (SSO), the Harvard University Origin of Life Initiative (HUOLI), the Scottish Universities Physics Alliance (SUPA), the University of Geneva, the Smithsonian Astrophysical Observatory (SAO), and the Italian National Astrophysical Institute (INAF), University of St. Andrews, Queens University Belfast, and University of Edinburgh. The research leading to these results has received funding from the European Union Seventh Framework Programme (FP7/2007-2013) under Grant Agreement No. 313014 (ETAEARTH). X. Dumusque would like to thank the Swiss National Science Foundation (SNSF) for its support through an Early Postdoc Mobility fel- lowship. P. Figueira acknowledges support by Fundação para a Ciência e a Tecnologia (FCT) through the Investigador FCT contract of reference IF/01037/2013 and POPH/FSE (EC) by FEDER funding through the program "Programa Operacional de Factores de Competitividade - COMPETE.” R. D. Haywood acknowledges support from an STFC postgraduate research studentship. This publication was made possible through the support of a grant from the John Templeton Foundation. The opinions expressed in this publication are those of the authors and do not necessarily reflect the views of the John Templeton Foundation. This research has made use of the results produced by the PI2S 2 Project managed by the Consorzio COMETA, a co-funded project by the Italian Ministero dellIstruzione, Università e Ricerca (MIUR) within the Piano Operativo Nazionale Ricerca Scientifica, Sviluppo Tecnologico, Alta Formazione (PON 20002006). We would like to thank A. McWilliam, I. Ivans, and C. Sneden for providing us their software that interpolates between atmospheric models.

\section{REFERENCES}

Adibekyan, V. Z., Figueira, P., Santos, N. C., et al. 2013, A\&A, 554, A44 Aigrain, S., Pont, F., \& Zucker, S. 2012, MNRAS, 419, 3147

Barnes, S. A. 2007, ApJ, 669, 1167

Batalha, N. M., Borucki, W. J., Bryson, S. T., et al. 2011, ApJ, 729, 27

Boisse, I., Bonfils, X., \& Santos, N. C. 2012, A\&A, 545, 109

Boisse, I., Bouchy, F., Hébrard, G., et al. 2011, A\&A, 528, A4

Boisse, I., Eggenberger, A., Santos, N. C., et al. 2010, A\&A, 523, A88

Boisse, I., Moutou, C., Vidal-Madjar, A., et al. 2009, A\&A, 495, 959

Bouchy, F., Hebb, L., Skillen, I., et al. 2010, A\&A, 519, A98

Buchhave, L. A., Latham, D. W., Johansen, A., et al. 2012, Natur, 486, 375

Buchhave, L. A., Bizzarro, M., Latham, D. W., et al. 2014, arXiv:1405.7695

Carter, J. A., Agol, E., Chaplin, W. J., et al. 2012, Sci, 337, 556

Castelli, F., \& Kurucz, R. L. 2004, arXivastro-ph/0405087

Cayrel, R., Depagne, E., Spite, M., et al. 2004, A\&A, 416, 1117

Chib, S., \& Jeliazkov, I. 2001, J. Am. Stat. Assoc., 96, 279

Cosentino, R., Lovis, C., Pepe, F., et al. 2012, SPIE, 8446, 84461V

Demarque, P., Woo, J.-H., Kim, Y.-C., \& Yi, S. K. 2004, ApJS, 155, 667

Dumusque, X., Pepe, F., Lovis, C., et al. 2012, Natur, 491, 207

Dumusque, X., Santos, N. C., Udry, S., Lovis, C., \& Bonfils, X. 2011a, A\&A, 527, A82

Dumusque, X., Udry, S., Lovis, C., Santos, N. C., \& Monteiro, M. J. P. F. G. 2011b, A\&A, 525, A140

Eastman, J., Gaudi, B. S., \& Agol, E. 2013, PASP, 125, 83

Eastman, J., Siverd, R., \& Gaudi, B. S. 2010, PASP, 122, 935

Elkins-Tanton, L. T. 2011, Ap\&SS, 332, 359

Everett, M. E., Howell, S. B., \& Kinemuchi, K. 2012, PASP, 124, 316

Feroz, F., \& Hobson, M. P. 2013, MNRAS, 437, 3540

Fogtmann-Schulz, A., Hinrup, B., Van Eylen, V., et al. 2014, ApJ, 781, 8

Ford, E. B. 2006, ApJ, 642, 505

Fressin, F., Torres, G., Désert, J.-M., et al. 2011, ApJS, 197, 5

Gelman, A., Carlin, J. B., Stern, H. S., \& Rubin, D. B. 2004, Bayesian Data Analysis (Boca Raton, FL: Chapman and Hall/CRC)

Gilliland, R. L., Marcy, G. W., Rowe, J. F., et al. 2013, ApJ, 766, 40

Giménez, A. 2006, A\&A, 450, 1231

Grasset, O., Schneider, J., \& Sotin, C. 2009, ApJ, 693, 722

Gregory, P. C. 2005, ApJ, 631, 1198

Hatzes, A. P., Dvorak, R., Wuchterl, G., et al. 2010, A\&A, 520, A93

Hirano, T., Sanchis-Ojeda, R., Takeda, Y., et al. 2014, ApJ, 783, 9

Hirano, T., Suto, Y., Taruya, A., et al. 2010, ApJ, 709, 458

Howard, A. W., Sanchis-Ojeda, R., Marcy, G. W., et al. 2013, Natur, 503, 381 Howard, R. 2000, in Sunspot Evolution, ed. P. Murdin (Bristol: IOP Publishing), Howell, S. B., Sobeck, C., Haas, M., et al. 2014, PASP, 126, 398

Ikoma, M., \& Hori, Y. 2012, ApJ, 753, 66

Jeffreys, S. H. 1961, in The Theory of Probability, (Oxford: Oxford University Press)

Jenkins, J. M., Caldwell, D. A., Chandrasekaran, H., et al. 2010, ApJL, 713, L87

Kipping, D. M. 2013, MNRAS, 435, 2152

Knutson, H. A., Charbonneau, D., Cowan, N. B., et al. 2009, ApJ, 703, 769

Kurucz, R. L. 1992, in IAU Symp. 149, The Stellar Populations of Galaxies, ed. B. Barbuy \& A. Renzini (Dordrecht: Kluwer), 225

Latham, D. W., Rowe, J. F., Quinn, S. N., et al. 2011, ApJL, 732, L24 
Léger, A., Rouan, D., Schneider, J., et al. 2009, A\&A, 506, 287

Lissauer, J. J., Fabrycky, D. C., Ford, E. B., et al. 2011, Natur, 470, 53

Lissauer, J. J., Jontof-Hutter, D., Rowe, J. F., et al. 2013, ApJ, 770, 131

Lopez, E. D., \& Fortney, J. J. 2013, ApJ, 776, 2

Mamajek, E. E., \& Hillenbrand, L. A. 2008, ApJ, 687, 1264

Marcus, R. A., Sasselov, D., Hernquist, L., \& Stewart, S. T. 2010, ApJ, 712,73

Marcy, G. W., Isaacson, H., Howard, A. W., et al. 2014, ApJS, 210, 20

Mayor, M., Pepe, F., Queloz, D., et al. 2003, Msngr, 114, 20

Mestel, L. 1968, MNRAS, 138, 359

Montesinos, B., Thomas, J. H., Ventura, P., \& Mazzitelli, I. 2001, MNRAS, 326, 877

Noyes, R. W., Hartmann, L. W., Baliunas, S. L., Duncan, D. K., \& Vaughan, A. H. 1984, ApJ, 279, 763

Pace, G., \& Pasquini, L. 2004, A\&A, 426, 1021

Pepe, F., Collier Cameron, A., Latham, D. W., et al. 2013, Natur, 503, 377

Queloz, D., Bouchy, F., Moutou, C., et al. 2009, A\&A, 506, 303

Rafikov, R. R. 2006, ApJ, 648, 666

Rauer, H., Catala, C., Aerts, C., et al. 2013, arXiv:1310.0696

Ricker, G. R., Latham, D. W., Vanderspek, R. K., et al. 2010, BAAS, 42, 215

Rogers, L. A., Bodenheimer, P., Lissauer, J. J., \& Seager, S. 2011, ApJ, 738, 59

Rowe, J. F., Matthews, J. M., Seager, S., et al. 2006, ApJ, 646, 1241

Santerne, A., Díaz, R. F., Bouchy, F., et al. 2011, A\&A, 528, A63
Santos, N. C., Mayor, M., Naef, D., et al. 2002, A\&A, 392, 215

Schatzman, E. 1962, AnAp, 25, 18

Sing, D. K. 2010, A\&A, 510, A21

Smith, J. C., Stumpe, M. C., Van Cleve, J. E., et al. 2012, PASP, 124, 1000

Sneden, C. 1973, ApJ, 184, 839

Soderblom, D. R., Duncan, D. K., \& Johnson, D. R. H. 1991, ApJ, 375, 722

Soubiran, C., \& Girard, P. 2005, A\&A, 438, 139

Sousa, S. G., Santos, N. C., Israelian, G., Mayor, M., \& Monteiro, M. J. P. F. G. 2007, A\&A, 469, 783

Sousa, S. G., Santos, N. C., Israelian, G., Mayor, M., \& Udry, S. 2011, A\&A, 533, A141

Sozzetti, A., Torres, G., Charbonneau, D., et al. 2007, ApJ, 664, 1190

Ter Braak, C. J. F. 2006, Stat. Comput., 16, 239

Torres, G., Fischer, D. A., Sozzetti, A., et al. 2012, ApJ, 757, 161

Torres, G., Fressin, F., Batalha, N. M., et al. 2011, ApJ, 727, 24

Valencia, D., Sasselov, D. D., \& O'Connell, R. J. 2007, ApJ, 665, 1413

Valenti, J. A., \& Piskunov, N. 1996, A\&AS, 118, 595

Weber, E. J., \& Davis, L., Jr. 1967, ApJ, 148, 217

Wilson, O. C. 1963, ApJ, 138, 832

Zacharias, N., Finch, C., Girard, T., et al. 2009, yCat, 1315, 0

Zechmeister, M., \& Kürster, M. 2009, A\&A, 496, 577

Zeng, L., \& Sasselov, D. 2013, PASP, 125, 227

Zeng, L., \& Sasselov, D. 2014, ApJ, 784, 96 